\title{
Molecular Tweezer and Clip in Aqueous Solution: Unexpected Self-Assembly, Powerful Host-Guest Complex Formation, Quantum Chemical ${ }^{1}$ H NMR Shift Calculation
}

Frank-Gerrit Klärner*, Björn Kahlert, Anke Nellesen, Jan Zienau, Christian Ochsenfeld*, Thomas Schrader*

\section{Supporting Information}

1. Optimization of the tweezer and clip structures by force-field methods and calculation of the chemical ${ }^{1} \mathrm{H}$ NMR shifts by quantum chemical methods

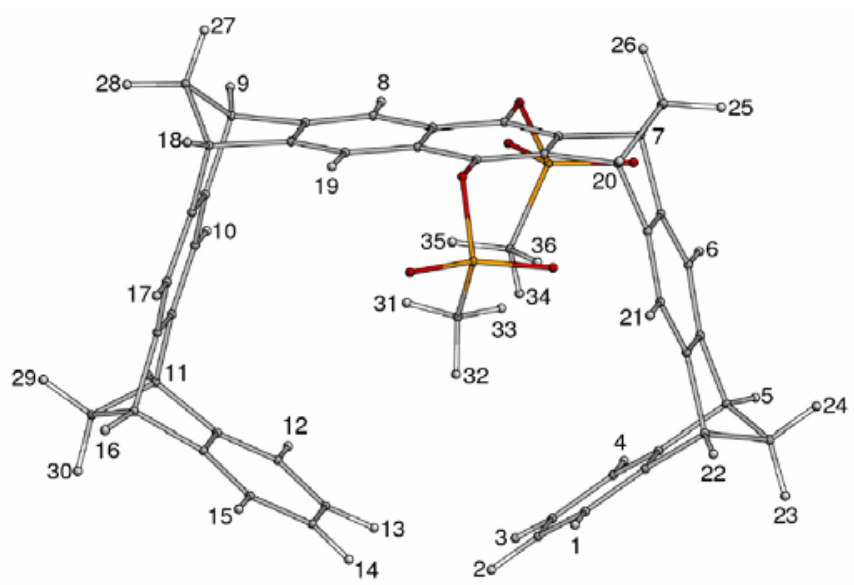

Structure E1 $\left(\mathrm{E}_{\mathrm{Amber} *}=52.9 \mathrm{kcal} / \mathrm{mol}\right)$

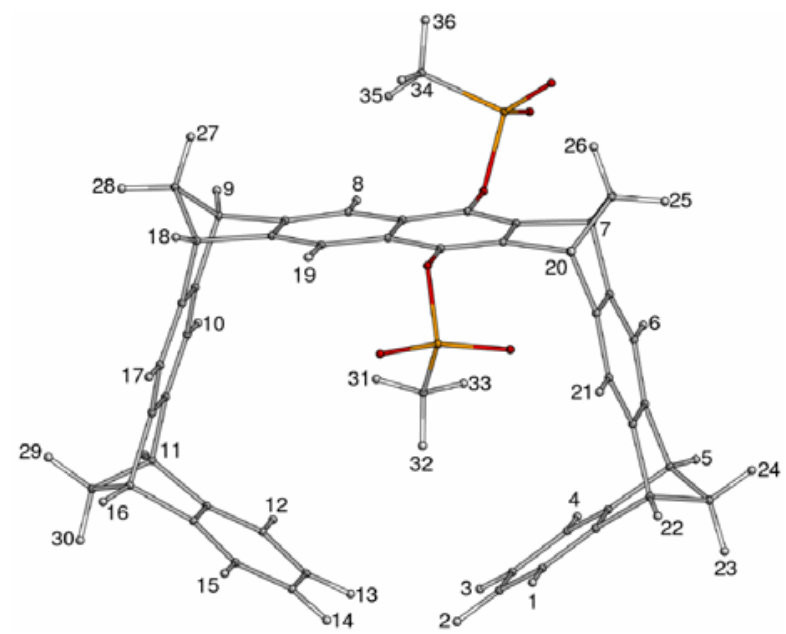

Structure E2 $\left(\mathrm{E}_{\mathrm{Amber} *}=53.4 \mathrm{kcal} / \mathrm{mol}\right)$ 


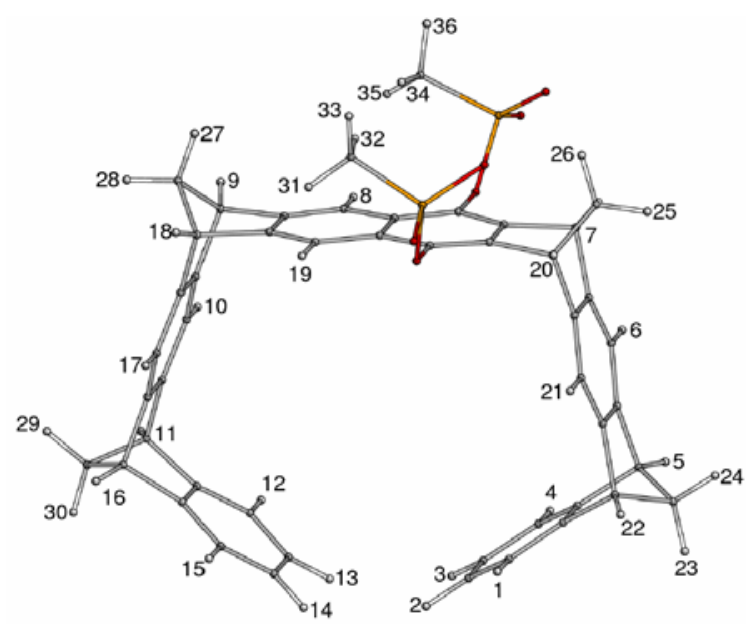

Structure E3 $\left(\mathrm{E}_{\mathrm{Amber} *}=53.7 \mathrm{kcal} / \mathrm{mol}\right)$

Figure S1: Structures of tweezer 2a calculated by a Monte Carlo Conformer Search (Macromodel 6.5, Amber*/ $\mathrm{H}_{2} \mathrm{O}, 5000$ structures).

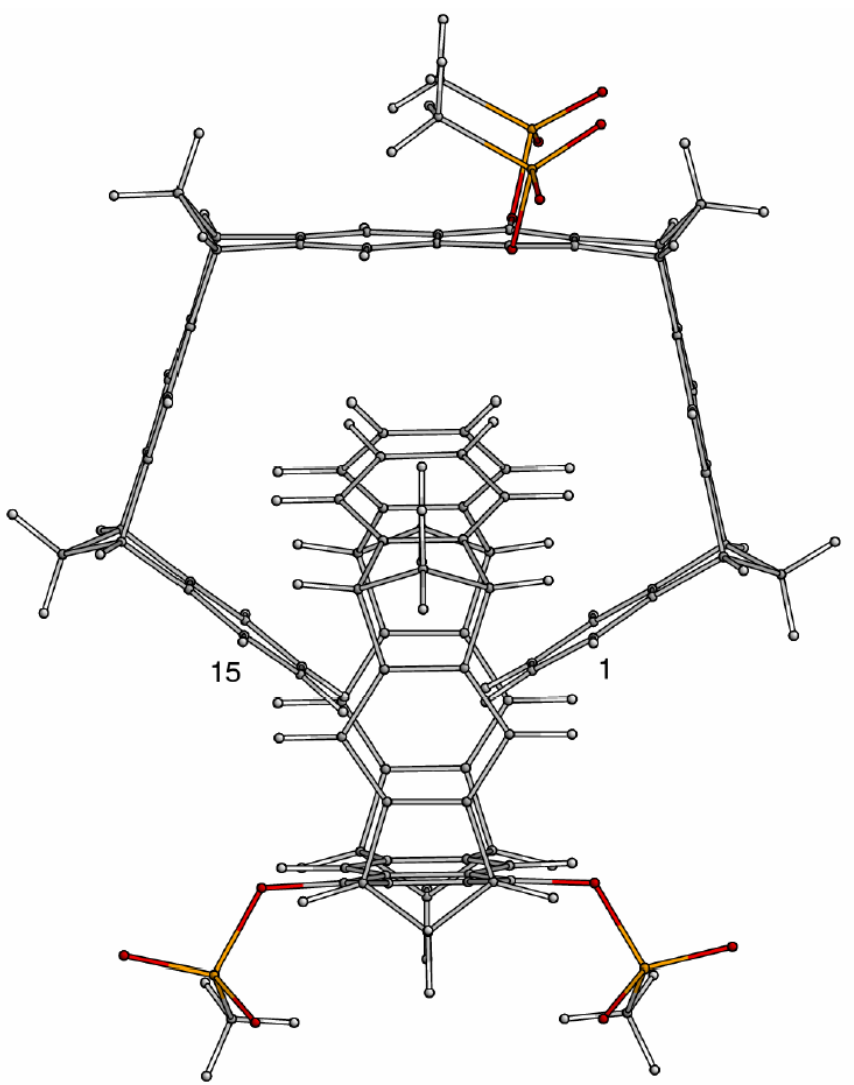

Figure S2: Structure of dimer $[2 \mathrm{a}]_{2}\left(\mathrm{E}_{\mathrm{Amber}}{ }^{*}=85.5 \mathrm{kcal} / \mathrm{mol}\right)$ calculated by a Monte Carlo Conformer Search (Macromodel 6.5, Amber*/ $\mathrm{H}_{2} \mathrm{O}, 5000$ structures). 
Table S1: Comparison of ${ }^{1} \mathrm{H}$ NMR shifts [ppm] of tweezer $2 \mathbf{a}\left(500 \mathrm{MHz}, 25^{\circ} \mathrm{C}, \mathrm{CD}_{3} \mathrm{OD}\right)$ with those calculated for structure E1 by quantum chemical methods. The geometry of 2a used for the quantum chemical calculations was optimized by a Monte Carlo Conformer Search (MacroModel 6.5, Amber ${ }^{*} / \mathrm{H}_{2} \mathrm{O}, 5000$ structures).

\begin{tabular}{|c|c|c|c|}
\hline Atom-Nr. & GIAO-HF/SVP & GIAO-HF/TZP & Exp. $\left(\mathrm{CD}_{3} \mathrm{OD}\right)$ \\
\hline 1 & 7.2 & 7.4 & \multirow{4}{*}{7.1} \\
\hline 4 & 7.2 & 7.4 & \\
\hline 12 & 7.2 & 7.4 & \\
\hline 15 & 7.2 & 7.4 & \\
\hline 2 & 6.7 & 6.9 & \multirow{4}{*}{6.7} \\
\hline 3 & 6.7 & 6.9 & \\
\hline 13 & 7.0 & 7.2 & \\
\hline 14 & 7.0 & 7.2 & \\
\hline 5 & 3.7 & 3.9 & \multirow{4}{*}{4.1} \\
\hline 22 & 3.7 & 3.9 & \\
\hline 11 & 3.6 & 3.8 & \\
\hline 16 & 3.6 & 3.8 & \\
\hline 6 & 8.6 & 8.8 & \multirow{2}{*}{7.4} \\
\hline 21 & 8.6 & 8.8 & \\
\hline 10 & 7.3 & 7.5 & \multirow{2}{*}{7.2} \\
\hline 17 & 7.3 & 7.5 & \\
\hline 7 & 5.2 & 5.4 & \multirow{2}{*}{4.8} \\
\hline 20 & 5.2 & 5.4 & \\
\hline 9 & 3.8 & 3.9 & \multirow{2}{*}{4.2} \\
\hline 18 & 3.7 & 3.9 & \\
\hline 8 & 8.2 & 8.3 & \multirow{2}{*}{7.9} \\
\hline 19 & 8.1 & 8.3 & \\
\hline 23 & 1.8 & 2.0 & \multirow{2}{*}{2.4} \\
\hline 24 & 2.1 & 2.2 & \\
\hline 25 & 1.5 & 1.7 & \multirow{2}{*}{2.3} \\
\hline 26 & 1.7 & 1.8 & \\
\hline 27 & 2.1 & 2.2 & \multirow{4}{*}{2.4} \\
\hline 28 & 1.8 & 2.0 & \\
\hline 29 & 2.0 & 2.2 & \\
\hline 30 & 2.0 & 2.1 & \\
\hline 31 & -1.1 & -1.1 & \multirow{6}{*}{1.2} \\
\hline 32 & -0.6 & -0.6 & \\
\hline 33 & -1.4 & -1.4 & \\
\hline 34 & -0.6 & -0.6 & \\
\hline 35 & -1.0 & -1.0 & \\
\hline 36 & -1.4 & -1.4 & \\
\hline
\end{tabular}


Table S2: Comparison of ${ }^{1} \mathrm{H}$ NMR shifts $[\mathrm{ppm}]$ of tweezer $\mathbf{2 a}\left(500 \mathrm{MHz}, 25^{\circ} \mathrm{C}, \mathrm{CD}_{3} \mathrm{OD}\right)$ with those calculated for structure E2 by quantum chemical methods. The geometry of 2a used for the quantum chemical calculations was optimized by a Monte Carlo Conformer Search (MacroModel 6.5, Amber ${ }^{*} / \mathrm{H}_{2} \mathrm{O}, 5000$ structures).

\begin{tabular}{|c|c|c|c|}
\hline Atom-Nr. & GIAO-HF/SVP & GIAO-HF/TZP & Exp. $\left(C_{3} \mathrm{OD}\right)$ \\
\hline 1 & 7.3 & 7.4 & \multirow{4}{*}{7.1} \\
\hline 4 & 7.1 & 7.3 & \\
\hline 12 & 7.2 & 7.4 & \\
\hline 15 & 7.3 & 7.5 & \\
\hline 2 & 6.8 & 7.0 & \multirow{4}{*}{6.7} \\
\hline 3 & 6.7 & 6.9 & \\
\hline 13 & 7.0 & 7.2 & \\
\hline 14 & 7.0 & 7.2 & \\
\hline 5 & 3.6 & 3.8 & \multirow{4}{*}{4.1} \\
\hline 22 & 3.7 & 3.9 & \\
\hline 11 & 3.6 & 3.7 & \\
\hline 16 & 3.6 & 3.8 & \\
\hline 6 & 7.3 & 7.5 & \multirow{2}{*}{7.4} \\
\hline 21 & 8.6 & 8.9 & \\
\hline 10 & 7.2 & 7.4 & \multirow{2}{*}{7.2} \\
\hline 17 & 7.3 & 7.5 & \\
\hline 7 & 5.3 & 5.4 & \multirow{2}{*}{4.8} \\
\hline 20 & 5.3 & 5.5 & \\
\hline 9 & 3.7 & 3.8 & \multirow{2}{*}{4.2} \\
\hline 18 & 3.8 & 4.0 & \\
\hline 8 & 7.8 & 7.9 & \multirow{2}{*}{7.9} \\
\hline 19 & 8.2 & 8.4 & \\
\hline 23 & 1.9 & 2.0 & \multirow{2}{*}{2.4} \\
\hline 24 & 2.1 & 2.2 & \\
\hline 25 & 1.6 & 1.7 & \multirow{2}{*}{2.3} \\
\hline 26 & 2.3 & 2.5 & \\
\hline 27 & 2.1 & 2.2 & \multirow{4}{*}{2.4} \\
\hline 28 & 1.8 & 1.9 & \\
\hline 29 & 2.1 & 2.2 & \\
\hline 30 & 2.0 & 2.1 & \\
\hline 31 & -1.1 & -1.1 & \multirow{6}{*}{1.2} \\
\hline 32 & -0.7 & -0.6 & \\
\hline 33 & -1.5 & -1.5 & \\
\hline 34 & 0.3 & 0.3 & \\
\hline 35 & 0.7 & 0.7 & \\
\hline 36 & 0.3 & 0.4 & \\
\hline
\end{tabular}


Table S3: Comparison of ${ }^{1} \mathrm{H}$ NMR shifts $[\mathrm{ppm}]$ of tweezer $2 \mathbf{a}\left(500 \mathrm{MHz}, 25^{\circ} \mathrm{C}, \mathrm{CD}_{3} \mathrm{OD}\right)$ with those calculated for structure E3 by quantum chemical methods. The geometry of 2a used for the quantum chemical calculations was optimized by a Monte Carlo Conformer Search (MacroModel 6.5, Amber ${ }^{*} / \mathrm{H}_{2} \mathrm{O}, 5000$ structures).

\begin{tabular}{|c|c|c|c|}
\hline Atom-Nr. & GIAO-HF/SVP & GIAO-HF/TZP & Exp. $\left(\mathrm{CD}_{3} \mathrm{OD}\right)$ \\
\hline 1 & 7.1 & 7.4 & \multirow{4}{*}{7.1} \\
\hline 4 & 7.1 & 7.4 & \\
\hline 12 & 7.2 & 7.4 & \\
\hline 15 & 7.2 & 7.5 & \\
\hline 2 & 6.7 & 7.0 & \multirow{4}{*}{6.7} \\
\hline 3 & 6.7 & 7.0 & \\
\hline 13 & 7.0 & 7.2 & \\
\hline 14 & 7.0 & 7.2 & \\
\hline 5 & 3.6 & 3.8 & \multirow{4}{*}{4.1} \\
\hline 22 & 3.6 & 3.8 & \\
\hline 11 & 3.6 & 3.8 & \\
\hline 16 & 3.6 & 3.8 & \\
\hline 6 & 7.4 & 7.6 & \multirow{2}{*}{7.4} \\
\hline 21 & 7.4 & 7.6 & \\
\hline 10 & 7.2 & 7.4 & \multirow{2}{*}{7.2} \\
\hline 17 & 7.2 & 7.4 & \\
\hline 7 & 5.3 & 5.5 & \multirow{2}{*}{4.8} \\
\hline 20 & 5.3 & 5.5 & \\
\hline 9 & 3.7 & 3.9 & \multirow{2}{*}{4.2} \\
\hline 18 & 3.7 & 3.9 & \\
\hline 8 & 7.9 & 8.0 & \multirow{2}{*}{7.9} \\
\hline 19 & 7.9 & 8.0 & \\
\hline 23 & 1.9 & 2.1 & \multirow{2}{*}{2.4} \\
\hline 24 & 2.1 & 2.3 & \\
\hline 25 & 1.6 & 1.8 & \multirow{2}{*}{2.3} \\
\hline 26 & 2.9 & 3.2 & \\
\hline 27 & 2.1 & 2.2 & \multirow{4}{*}{2.4} \\
\hline 28 & 1.7 & 1.9 & \\
\hline 29 & 2.0 & 2.2 & \\
\hline 30 & 2.0 & 2.2 & \\
\hline 31 & 0.6 & 0.6 & \multirow{6}{*}{1.2} \\
\hline 32 & 0.3 & 0.3 & \\
\hline 33 & 0.4 & 0.4 & \\
\hline 34 & 0.3 & 0.3 & \\
\hline 35 & 0.6 & 0.6 & \\
\hline 36 & 0.3 & 0.4 & \\
\hline
\end{tabular}


Table S4: Comparison of ${ }^{1} \mathrm{H}$ NMR shifts [ppm] of tweezer $2 \mathrm{a}\left(500 \mathrm{MHz}, 25^{\circ} \mathrm{C}, \mathrm{D}_{2} \mathrm{O}\right)$ with those calculated for dimer $[\mathbf{2} \mathbf{a}]_{2}$ by quantum chemical methods. The geometry of $[\mathbf{2 a}]_{2}$ used for the quantum chemical calculations was optimized by a Monte Carlo Conformer Search (MacroModel 6.5, Amber ${ }^{*} / \mathrm{H}_{2} \mathrm{O}, 5000$ structures).

\begin{tabular}{|c|c|c|c|}
\hline Atom-Nr. & GIAO-HF/SVP & GIAO-HF/TZP & Exp. $\left(D_{2} O\right)$ \\
\hline 1 & 6.6 & 6.8 & \multirow{4}{*}{$6.3 / 6.4$} \\
\hline 4 & 6.3 & 6.5 & \\
\hline 12 & 6.5 & 6.7 & \\
\hline 15 & 6.8 & 7.0 & \\
\hline 2 & 3.4 & 3.5 & \multirow{4}{*}{$3.6 / 3.7$} \\
\hline 3 & 3.2 & 3.3 & \\
\hline 13 & 4.0 & 4.1 & \\
\hline 14 & 4.3 & 4.4 & \\
\hline 5 & 3.5 & 3.7 & \multirow{4}{*}{4.3} \\
\hline 22 & 3.7 & 4.0 & \\
\hline 11 & 3.6 & 3.8 & \\
\hline 16 & 3.8 & 4.0 & \\
\hline 6 & 7.5 & 7.8 & \multirow{2}{*}{7.6} \\
\hline 21 & 7.6 & 7.9 & \\
\hline 10 & 7.5 & 7.7 & \multirow{2}{*}{7.5} \\
\hline 17 & 7.5 & 7.7 & \\
\hline 7 & 5.2 & 5.4 & \multirow{2}{*}{4.7} \\
\hline 20 & 5.2 & 5.4 & \\
\hline 9 & 3.6 & 3.7 & \multirow{2}{*}{4.4} \\
\hline 18 & 3.6 & 3.8 & \\
\hline 8 & 7.6 & 7.7 & \multirow{2}{*}{7.4} \\
\hline 19 & 7.5 & 7.6 & \\
\hline 23 & 2.3 & 2.5 & \multirow{2}{*}{2.5} \\
\hline 24 & 1.9 & 2.1 & \\
\hline 25 & 1.6 & 1.8 & \multirow{2}{*}{2.6} \\
\hline 26 & 2.9 & 3.1 & \\
\hline 27 & 1.9 & 2.1 & \multirow{4}{*}{2.5} \\
\hline 28 & 1.7 & 1.9 & \\
\hline 29 & 1.9 & 2.1 & \\
\hline 30 & 2.3 & 2.5 & \\
\hline 31 & 0.4 & 0.4 & \multirow{6}{*}{1.3} \\
\hline 32 & 0.2 & 0.1 & \\
\hline 33 & 0.0 & 0.0 & \\
\hline 34 & 0.2 & 0.2 & \\
\hline 35 & 0.4 & 0.4 & \\
\hline 36 & 0.0 & 0.1 & \\
\hline
\end{tabular}




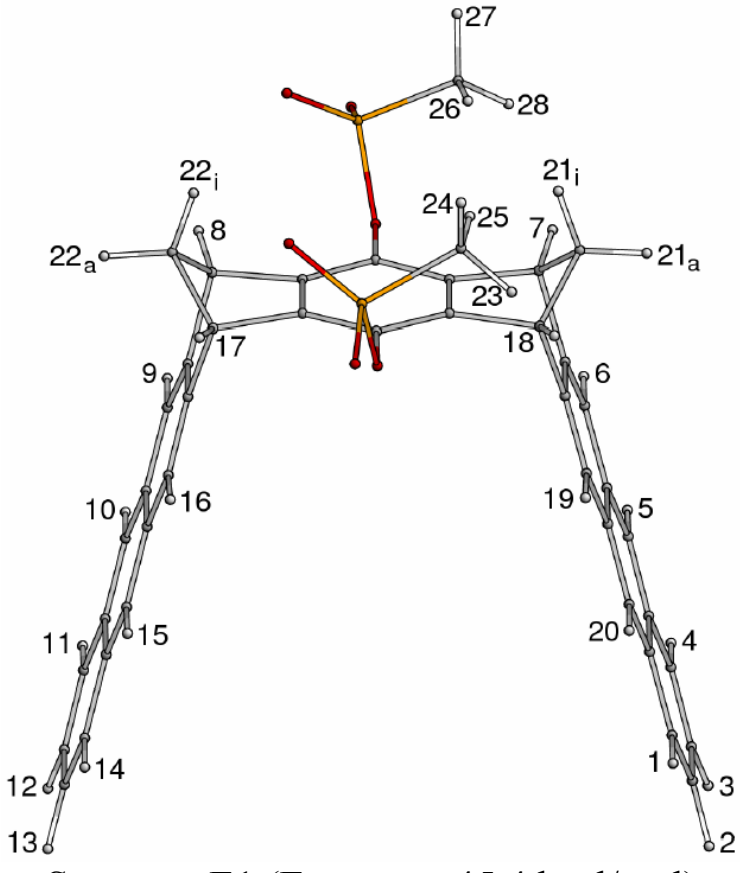

Structure E1 $\left(\mathrm{E}_{\text {Amber* }}=-45.4 \mathrm{kcal} / \mathrm{mol}\right)$

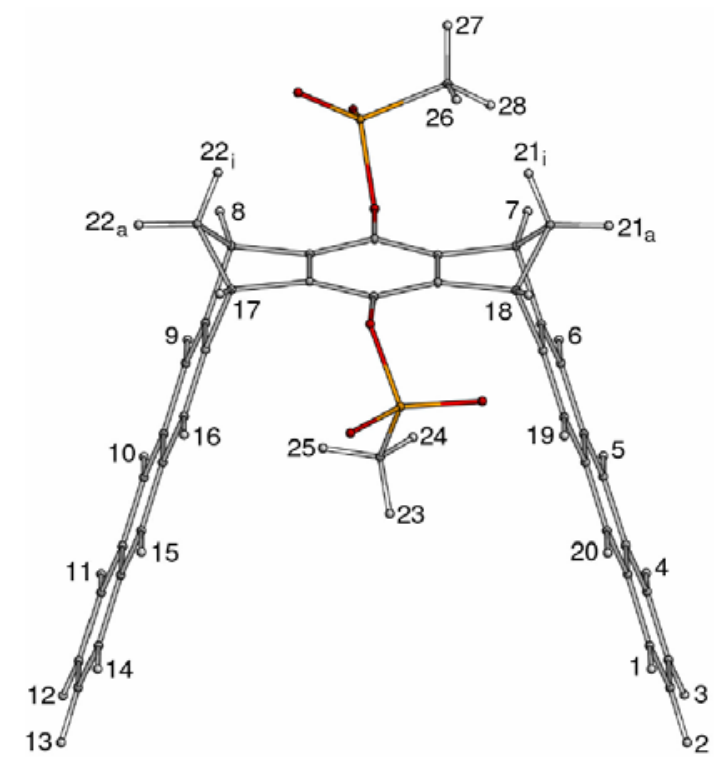

Structure E3 $\left(\mathrm{E}_{\text {Amber* }}=-45.6 \mathrm{kcal} / \mathrm{mol}\right)$

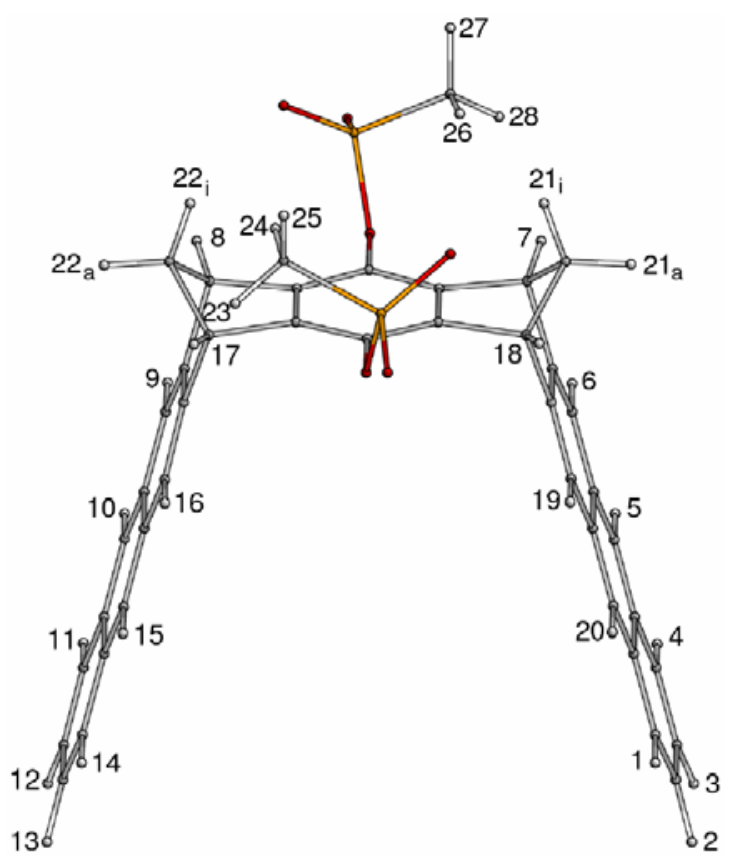

Structure E2 $\left(\mathrm{E}_{\text {Amber* }}=-45.5 \mathrm{kcal} / \mathrm{mol}\right)$

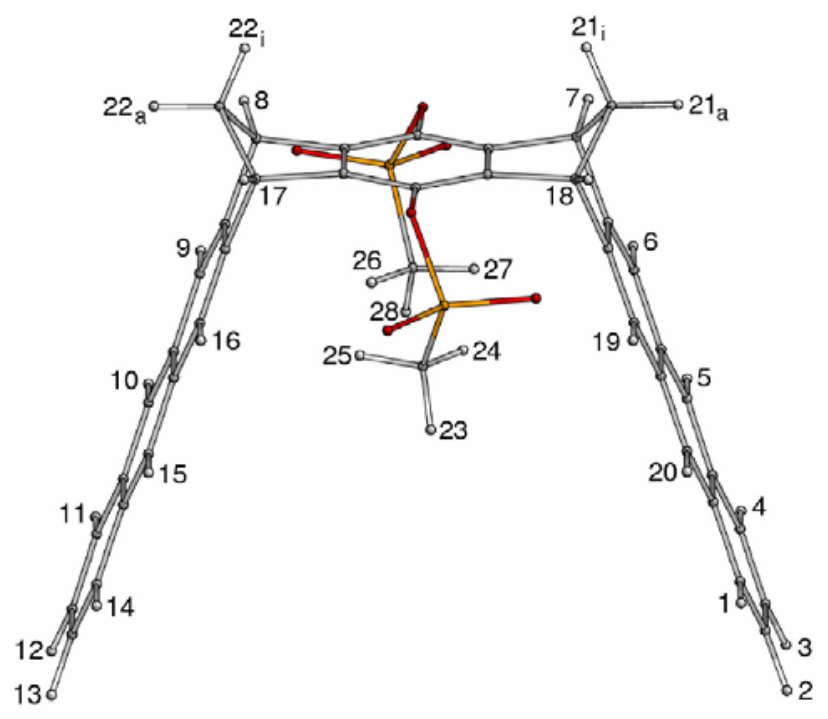

Structure E4 $\left(\mathrm{E}_{\text {Amber* }}=-46.2 \mathrm{kcal} / \mathrm{mol}\right)$

Figure S3: Structures of clip 4a calculated by a Monte Carlo Conformer Search (Macromodel 6.5, Amber* $/ \mathrm{H}_{2} \mathrm{O}, 5000$ structures). 


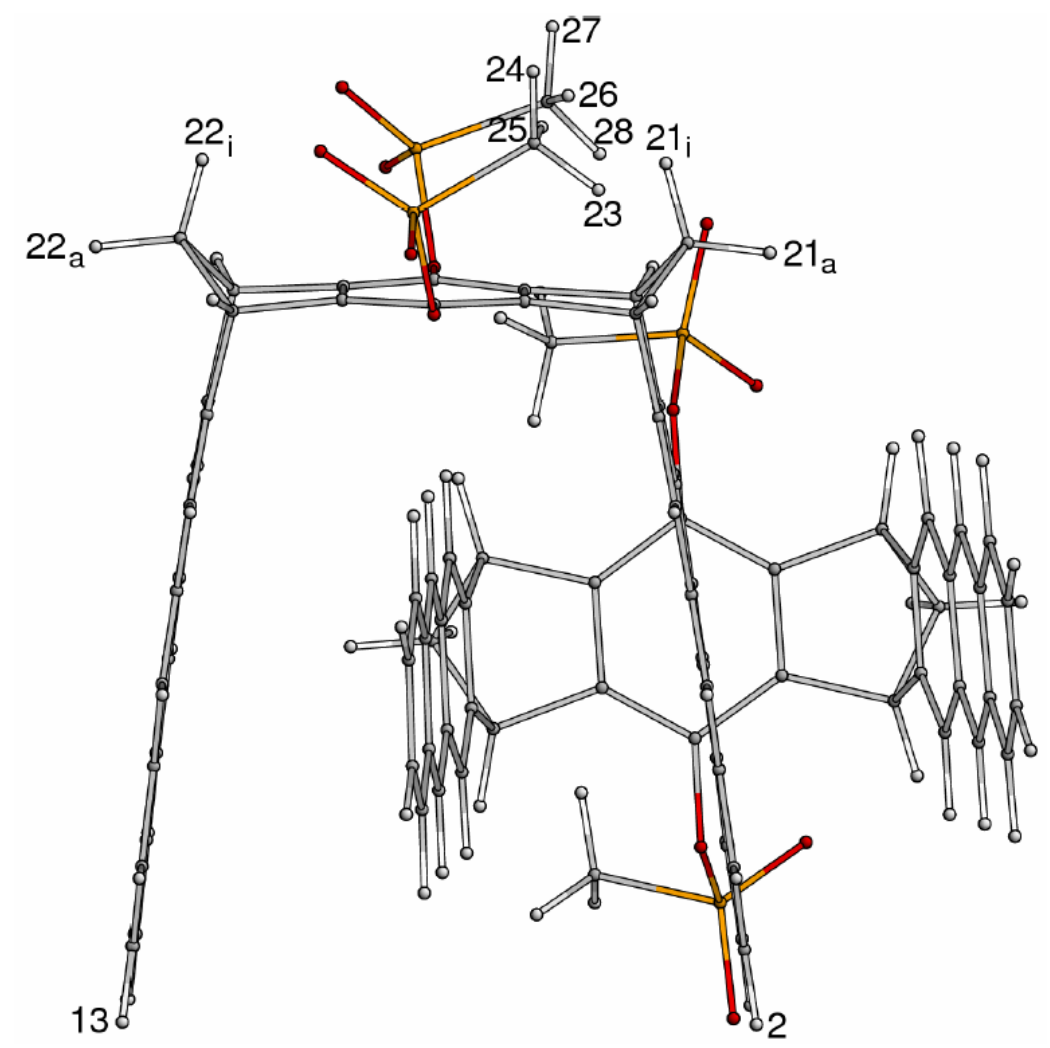

Figure S4: Structure of dimer $[\mathbf{4 a}]_{2}\left(\mathrm{E}_{\mathrm{Amber}^{*}}=-116.8 \mathrm{kcal} / \mathrm{mol}\right)$ calculated by a Monte Carlo Conformer Search (Macromodel 6.5, Amber*/ $\mathrm{H}_{2} \mathrm{O}, 5000$ structures). 
Table S5: Comparison of ${ }^{1} \mathrm{H}$ NMR shifts [ppm] of clip 4a $\left(500 \mathrm{MHz}, 25^{\circ} \mathrm{C}, \mathrm{CD}_{3} \mathrm{OD}\right)$ with those calculated for structure E1 by quantum chemical methods. The geometry of 4a used for the quantum chemical calculations was optimized by a Monte Carlo Conformer Search (MacroModel 6.5, Amber ${ }^{*} / \mathrm{H}_{2} \mathrm{O}, 5000$ structures).

\begin{tabular}{|c|c|c|c|}
\hline Atom-Nr. & GIAO-HF/SVP & GIAO-HF/TZP & Exp. $\left(C_{3} \mathrm{OD}\right)$ \\
\hline 1 & 7.7 & 7.9 & \multirow{4}{*}{7.8} \\
\hline 4 & 7.7 & 7.9 & \\
\hline 11 & 7.7 & 7.9 & \\
\hline 14 & 7.7 & 7.9 & \\
\hline 2 & 6.7 & 7.0 & \multirow{4}{*}{7.2} \\
\hline 3 & 6.7 & 7.0 & \\
\hline 12 & 6.6 & 6.8 & \\
\hline 13 & 6.6 & 6.8 & \\
\hline 5 & 8.1 & 8.3 & \multirow{4}{*}{8.1} \\
\hline 20 & 8.1 & 8.3 & \\
\hline 10 & 8.0 & 8.3 & \\
\hline 15 & 8.0 & 8.3 & \\
\hline 6 & 7.7 & 7.9 & \multirow{4}{*}{7.7} \\
\hline 19 & 7.7 & 7.9 & \\
\hline 9 & 7.8 & 8.0 & \\
\hline 16 & 7.8 & 8.0 & \\
\hline 7 & 4.4 & 4.6 & \multirow{4}{*}{4.8} \\
\hline 18 & 4.4 & 4.6 & \\
\hline 8 & 5.4 & 5.6 & \\
\hline 17 & 5.4 & 5.6 & \\
\hline $21 i$ & 2.3 & 2.4 & 2.6 \\
\hline $21 a$ & 1.7 & 1.8 & 2.3 \\
\hline $22 \mathrm{i}$ & 3.0 & 3.2 & 2.6 \\
\hline $22 a$ & 1.7 & 1.8 & 2.3 \\
\hline 23 & 0.8 & 0.8 & \multirow{6}{*}{1.4} \\
\hline 24 & 0.7 & 0.7 & \\
\hline 25 & 0.9 & 0.9 & \\
\hline 26 & 0.9 & 0.9 & \\
\hline 27 & 0.7 & 0.7 & \\
\hline 28 & 0.9 & 0.8 & \\
\hline
\end{tabular}


Table S6: Comparison of ${ }^{1} \mathrm{H}$ NMR shifts [ppm] of clip 4a $\left(500 \mathrm{MHz}, 25^{\circ} \mathrm{C}, \mathrm{CD}_{3} \mathrm{OD}\right)$ with those calculated for structure E2 by quantum chemical methods. The geometry of 4a used for the quantum chemical calculations was optimized by a Monte Carlo Conformer Search (MacroModel 6.5, Amber ${ }^{*} / \mathrm{H}_{2} \mathrm{O}, 5000$ structures).

\begin{tabular}{cccc}
\hline \hline Atom-Nr. & GIAO-HF/SVP & GIAO-HF/TZP & Exp. (CD OD $)$ \\
\hline \hline 1 & 7.7 & 7.9 & \\
4 & 7.7 & 7.9 & 7.8 \\
11 & 7.7 & 7.9 & \\
14 & 7.7 & 7.9 & \\
2 & 6.7 & 6.9 & 7.2 \\
3 & 6.7 & 6.9 & \\
12 & 6.7 & 6.9 & \\
13 & 6.7 & 6.9 & 8.1 \\
5 & 8.0 & 8.3 & \\
20 & 8.1 & 8.3 & \\
10 & 8.1 & 8.3 & 7.7 \\
15 & 8.0 & 8.3 & \\
6 & 7.7 & 7.8 & \\
19 & 7.8 & 8.0 & 4.8 \\
9 & 7.8 & 8.0 & \\
16 & 7.7 & 7.9 & \\
7 & 4.4 & 4.5 & \\
18 & 5.5 & 5.6 & \\
8 & 5.5 & 5.7 & \\
17 & 4.4 & 4.5 & \\
$21 \mathrm{i}$ & 2.7 & 2.8 & \\
$21 \mathrm{a}$ & 1.7 & 1.8 & \\
$22 \mathrm{i}$ & 2.7 & 2.8 & \\
$22 \mathrm{a}$ & 1.6 & 1.8 & \\
\hline 23 & 0.9 & 0.9 & \\
24 & 1.0 & 1.1 & \\
25 & 0.7 & 0.7 & \\
26 & 1.0 & 1.0 & \\
27 & 0.7 & 0.7 & \\
28 & 0.9 & 0.9 & \\
\hline \hline
\end{tabular}


Table S7: Comparison of ${ }^{1} \mathrm{H}$ NMR shifts [ppm] of clip 4a $\left(500 \mathrm{MHz}, 25^{\circ} \mathrm{C}, \mathrm{CD}_{3} \mathrm{OD}\right)$ with those calculated for structure E3 by quantum chemical methods. The geometry of 4a used for the quantum chemical calculations was optimized by a Monte Carlo Conformer Search (MacroModel 6.5, Amber ${ }^{*} / \mathrm{H}_{2} \mathrm{O}, 5000$ structures).

\begin{tabular}{|c|c|c|c|}
\hline Atom-Nr. & GIAO-HF/SVP & GIAO-HF/TZP & Exp. $\left(\mathrm{CD}_{3} \mathrm{OD}\right)$ \\
\hline 1 & 7.8 & 8.0 & \multirow{4}{*}{7.8} \\
\hline 4 & 7.7 & 7.9 & \\
\hline 11 & 7.7 & 7.9 & \\
\hline 14 & 7.8 & 8.0 & \\
\hline 2 & 6.6 & 6.9 & \multirow{4}{*}{7.2} \\
\hline 3 & 6.7 & 6.9 & \\
\hline 12 & 6.7 & 6.9 & \\
\hline 13 & 6.7 & 6.9 & \\
\hline 5 & 8.0 & 8.3 & \multirow{4}{*}{8.1} \\
\hline 20 & 8.3 & 8.5 & \\
\hline 10 & 8.1 & 8.3 & \\
\hline 15 & 8.2 & 8.4 & \\
\hline 6 & 7.6 & 7.8 & \multirow{4}{*}{7.7} \\
\hline 19 & 9.1 & 9.3 & \\
\hline 9 & 7.8 & 8.0 & \\
\hline 16 & 8.0 & 8.1 & \\
\hline 7 & 4.4 & 4.5 & \multirow{4}{*}{4.8} \\
\hline 18 & 5.7 & 5.9 & \\
\hline 8 & 5.5 & 5.6 & \\
\hline 17 & 4.6 & 4.7 & \\
\hline $21 i$ & 2.0 & 2.2 & 2.6 \\
\hline $21 a$ & 1.6 & 1.7 & 2.3 \\
\hline $22 \mathrm{i}$ & 2.6 & 2.7 & 2.6 \\
\hline $22 a$ & 1.7 & 1.8 & 2.3 \\
\hline 23 & -0.3 & -0.3 & \multirow{6}{*}{1.4} \\
\hline 24 & -1.1 & -1.1 & \\
\hline 25 & -0.3 & -0.3 & \\
\hline 26 & 0.9 & 0.9 & \\
\hline 27 & 0.6 & 0.7 & \\
\hline 28 & 1.0 & 1.0 & \\
\hline
\end{tabular}


Table S8: Comparison of ${ }^{1} \mathrm{H}$ NMR shifts [ppm] of clip 4a $\left(500 \mathrm{MHz}, 25^{\circ} \mathrm{C}, \mathrm{CD}_{3} \mathrm{OD}\right)$ with those calculated for structure E4 by quantum chemical methods. The geometry of 4a used for the quantum chemical calculations was optimized by a Monte Carlo Conformer Search (MacroModel 6.5, Amber ${ }^{*} / \mathrm{H}_{2} \mathrm{O}, 5000$ structures).

\begin{tabular}{cccc}
\hline Atom-Nr. & GIAO-HF/SVP & GIAO-HF/TZP & Exp. $\left(\mathrm{CD}_{3} \mathrm{OD}\right)$ \\
\hline 1 & 7.8 & 8.0 & \\
4 & 7.8 & 8.0 & 7.8 \\
11 & 7.8 & 8.0 & \\
14 & 7.8 & 7.9 & \\
2 & 6.7 & 6.9 & 7.2 \\
3 & 6.7 & 6.9 & \\
12 & 6.6 & 6.9 & \\
13 & 6.7 & 6.9 & 8.1 \\
5 & 8.2 & 8.4 & \\
20 & 8.3 & 8.5 & \\
10 & 8.3 & 8.5 & \\
15 & 8.2 & 8.4 & \\
6 & 7.9 & 8.1 & \\
19 & 9.1 & 9.3 & 4.8 \\
9 & 9.1 & 9.3 & \\
16 & 7.9 & 8.1 & \\
7 & 4.6 & 4.7 & \\
18 & 5.7 & 5.8 & 2.6 \\
8 & 5.7 & 5.9 & 2.3 \\
17 & 4.6 & 4.8 & \\
$21 \mathrm{i}$ & 1.9 & 2.1 & \\
$21 \mathrm{a}$ & 1.6 & 1.8 & \\
$22 \mathrm{i}$ & 1.9 & 2.1 & \\
$22 \mathrm{a}$ & 1.6 & 1.8 & \\
23 & -0.2 & -0.2 & \\
24 & -1.0 & -1.0 & \\
25 & -0.3 & -0.3 & \\
26 & -1.0 & -1.0 & \\
27 & -0.3 & -0.2 & \\
28 & -0.2 & & \\
\hline
\end{tabular}


Table S9: Comparison of ${ }^{1} \mathrm{H}$ NMR shifts [ppm] of clip 4a $\left(500 \mathrm{MHz}, 25^{\circ} \mathrm{C}, \mathrm{D}_{2} \mathrm{O}\right)$ with those calculated for dimer $[\mathbf{4 a}]_{2}$ by quantum chemical methods. The geometry of $[\mathbf{4 a}]_{2}$ used for the quantum chemical calculations was optimized by a Monte Carlo Conformer Search (MacroModel 6.5, Amber ${ }^{*} / \mathrm{H}_{2} \mathrm{O}, 5000$ structures).

\begin{tabular}{|c|c|c|c|}
\hline Atom-Nr. & GIAO-HF/SVP & GIAO-HF/TZP & Exp. $\left(\mathrm{D}_{2} \mathrm{O}\right)$ \\
\hline 1 & 5.9 & 6.1 & \multirow{4}{*}{6.8} \\
\hline 4 & 7.3 & 7.4 & \\
\hline 11 & 6.9 & 7.1 & \\
\hline 14 & 6.6 & 6.8 & \\
\hline 2 & 6.2 & 6.4 & \multirow{4}{*}{6.8} \\
\hline 3 & 6.7 & 7.0 & \\
\hline 12 & 6.2 & 6.4 & \\
\hline 13 & 6.1 & 6.3 & \\
\hline 5 & 2.5 & 2.6 & \multirow{4}{*}{5.8} \\
\hline 20 & 4.4 & 4.5 & \\
\hline 10 & 6.4 & 6.6 & \\
\hline 15 & 5.8 & 6.0 & \\
\hline 6 & 6.8 & 6.9 & \multirow{4}{*}{6.9} \\
\hline 19 & 5.9 & 6.0 & \\
\hline 9 & 7.2 & 7.4 & \\
\hline 16 & 6.8 & 7.0 & \\
\hline 7 & 5.3 & 5.5 & \multirow{4}{*}{4.7} \\
\hline 18 & 4.1 & 4.3 & \\
\hline 8 & 5.3 & 5.4 & \\
\hline 17 & 5.2 & 5.3 & \\
\hline $21 \mathrm{i}$ & 2.4 & 2.4 & 2.8 \\
\hline $21 a$ & 1.9 & 2.1 & 2.5 \\
\hline $22 \mathrm{i}$ & 3.1 & 3.3 & 2.8 \\
\hline $22 \mathrm{a}$ & 1.6 & 1.7 & 2.5 \\
\hline 23 & 1.0 & 0.9 & \multirow{6}{*}{1.8} \\
\hline 24 & 0.5 & 0.5 & \\
\hline 25 & 1.3 & 1.3 & \\
\hline 26 & 0.8 & 0.9 & \\
\hline 27 & 0.8 & 0.9 & \\
\hline 28 & 2.9 & 3.0 & \\
\hline
\end{tabular}


2. Determination of the association constants, $K_{\operatorname{dim}}$ or $K_{a}$, and the complexation-induced ${ }^{1} \mathrm{H}$ NMR shifts, $\Delta \delta_{\max }$, by the ${ }^{1} \mathrm{H}$ NMR dilution titration experiments at variable temperature

Table S10: ${ }^{1} \mathrm{H}$ NMR dilution titration experiments at variable temperatures to determine the association constants $K_{\text {dim }}$ and the differences in the ${ }^{1} \mathrm{H}$ NMR chemical shifts for the equilibrium $2 \cdot \mathbf{2 a} \leftrightarrows[2 \mathbf{a}]_{2}$ in $\mathrm{D}_{2} \mathrm{O}$

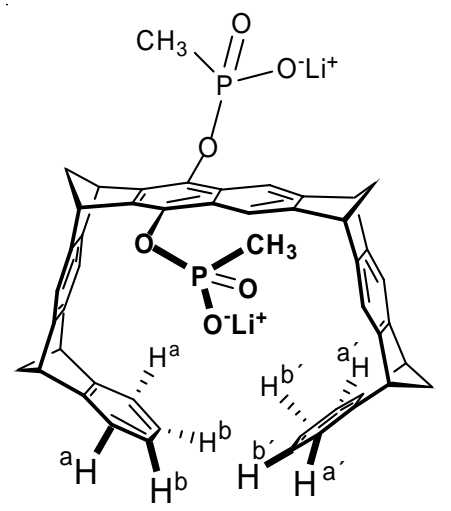

$$
\begin{array}{llr} 
& M\left[\mathrm{~g} \mathrm{~mol}^{-1}\right] & 784.64 \\
\delta \text { (monomer) } \mathrm{H}^{\mathrm{a}} \text { and } \mathrm{H}^{\mathrm{a}^{\prime}}[\mathrm{ppm}]=7.1^{*} & m[\mathrm{mg}] & 6.19 \\
\delta \text { (monomer) } \mathrm{H}^{\mathrm{b}} \text { and } \mathrm{H}^{\mathrm{b}^{\prime}}[\mathrm{ppm}]=6.7^{*} & V_{0}[\mathrm{~mL}] & 1.00 \\
& {[2 \mathrm{a}]_{0}[\mathrm{mM}]} & 7.90
\end{array}
$$

* The $\delta$ values observed in the ${ }^{1} \mathrm{H}$ NMR spectrum of $2 \mathbf{a}$ in $\mathrm{CD}_{3} \mathrm{OD}$ are used for $\delta$ (monomer).

$T[\mathrm{~K}]: 338$

\begin{tabular}{cccc}
\hline$[\mathbf{2 a}]_{0}[\mathrm{M}]$ & $\delta_{\text {obs }}\left(\mathrm{H}^{\mathrm{a}}\right)$ & $\Delta \delta_{\text {obs }}\left(\mathrm{H}^{\mathrm{a}}\right)$ & $\Delta \delta_{\text {calc }}\left(\mathrm{H}^{\mathrm{a}}\right)$ \\
\hline 0.00395 & 6.259 & 0.841 & 0.836 \\
0.00279 & 6.272 & 0.828 & 0.827 \\
0.00210 & 6.288 & 0.812 & 0.818 \\
0.00163 & 6.293 & 0.807 & 0.810 \\
0.00026 & 6.382 & 0.718 & 0.707 \\
0.00015 & 6.451 & 0.650 & 0.657 \\
\hline
\end{tabular}

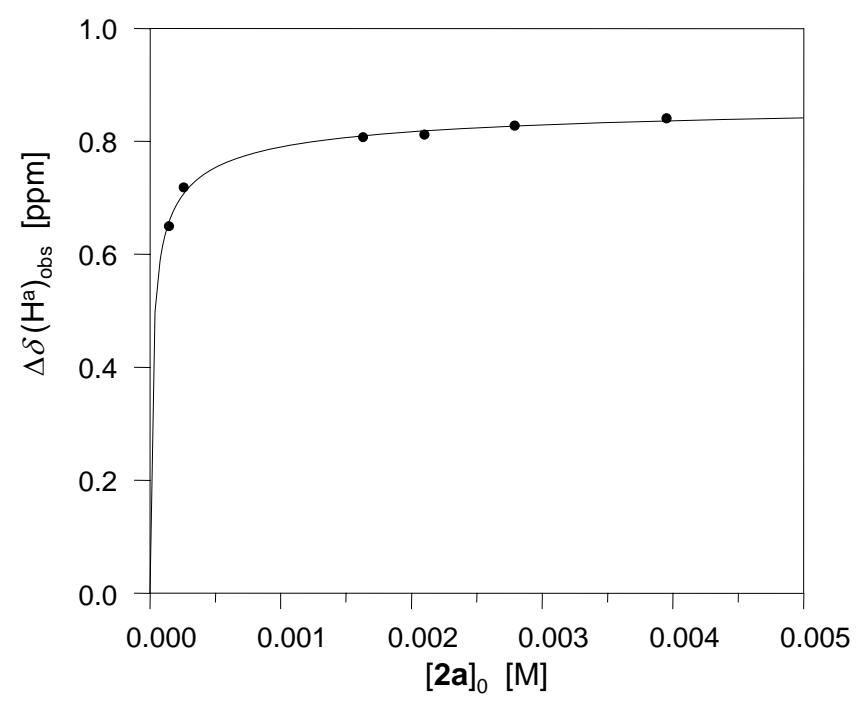

$$
\begin{array}{lll}
K_{\operatorname{dim}}\left[\mathrm{M}^{-1}\right]= & 38200 \pm 4 & 100 \\
\Delta \delta_{\max }\left(\mathrm{H}^{\mathrm{a}}\right)[\mathrm{ppm}] & = & 0.9 \\
\Delta \delta_{\max }\left(\mathrm{H}^{\mathrm{a}^{\prime}}\right)[\mathrm{ppm}] & = & 0.7 \\
\Delta \delta_{\max }\left(\mathrm{H}^{\mathrm{b}}\right)[\mathrm{ppm}] & = & 3.1 \\
\Delta \delta_{\max }\left(\mathrm{H}^{\mathrm{b}^{\prime}}\right)[\mathrm{ppm}]= & 2.8
\end{array}
$$

$$
[2 \mathrm{a}]_{0}[\mathrm{M}]
$$


$T[\mathrm{~K}]: 348$

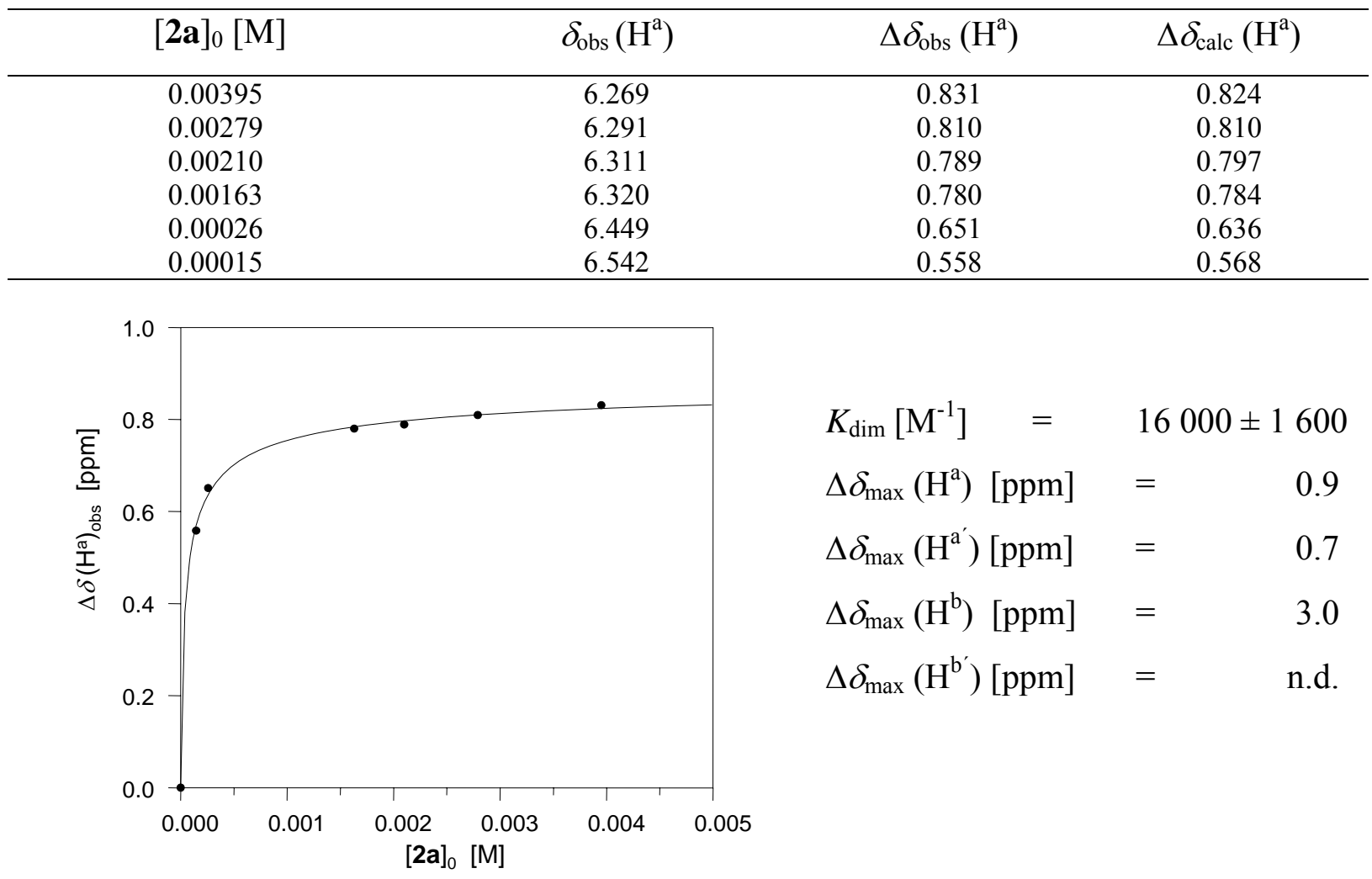

$T$ [K]: 358

\begin{tabular}{cccc}
\hline$[\mathbf{2 a}]_{0}[\mathrm{M}]$ & $\delta_{\text {obs }}\left(\mathrm{H}^{\mathrm{a}}\right)$ & $\Delta \delta_{\text {obs }}\left(\mathrm{H}^{\mathrm{a}}\right)$ & $\Delta \delta_{\text {calc }}\left(\mathrm{H}^{\mathrm{a}}\right)$ \\
\hline 0.00395 & 6.291 & 0.809 & 0.798 \\
0.00279 & 6.322 & 0.778 & 0.778 \\
0.00210 & 6.352 & 0.748 & 0.760 \\
0.00163 & 6.363 & 0.737 & 0.741 \\
0.00026 & 6.538 & 0.562 & 0.545 \\
0.00015 & 6.650 & 0.450 & 0.461 \\
\hline
\end{tabular}

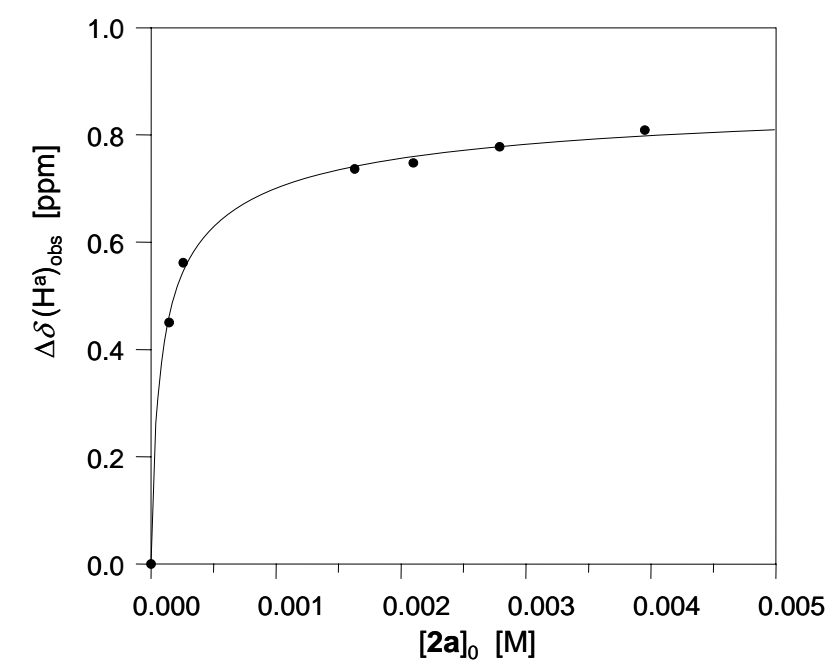

$K_{\operatorname{dim}}\left[\mathrm{M}^{-1}\right]=7200 \pm 700$

$\Delta \delta_{\max }\left(\mathrm{H}^{\mathrm{a}}\right)[\mathrm{ppm}]=0.9$

$\Delta \delta_{\max }\left(\mathrm{H}^{\mathrm{a}^{\prime}}\right)[\mathrm{ppm}]=0.7$

$\Delta \delta_{\max }\left(\mathrm{H}^{\mathrm{b}}\right)[\mathrm{ppm}]=$ n.d.

$\Delta \delta_{\max }\left(\mathrm{H}^{\mathrm{b}^{\prime}}\right)[\mathrm{ppm}]=2.6$ 
$T[\mathrm{~K}]: 368$

\begin{tabular}{cccc}
\hline$[\mathbf{2 a}]_{0}[\mathrm{M}]$ & $\delta_{\text {obs }}\left(\mathrm{H}^{\mathrm{a}}\right)$ & $\Delta \delta_{\text {obs }}\left(\mathrm{H}^{\mathrm{a}}\right)$ & $\Delta \delta_{\text {calc }}\left(\mathrm{H}^{\mathrm{a}}\right)$ \\
\hline 0.00395 & 6.327 & 0.773 & 0.755 \\
0.00279 & 6.376 & 0.724 & 0.728 \\
0.00210 & 6.413 & 0.687 & 0.702 \\
0.00163 & 6.429 & 0.671 & 0.677 \\
0.00026 & 6.653 & 0.447 & 0.431 \\
0.00015 & 6.769 & 0.331 & 0.340 \\
\hline
\end{tabular}

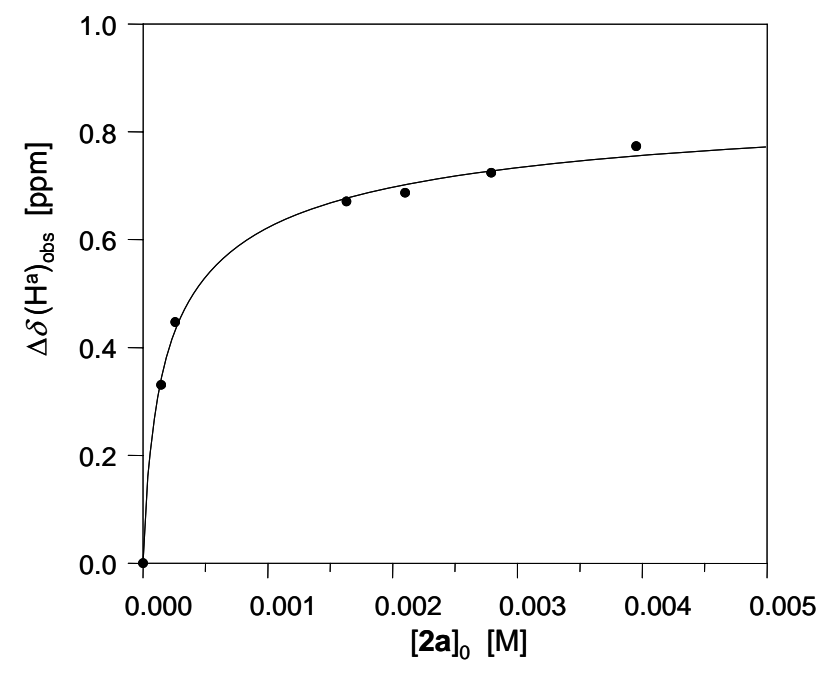

$\begin{array}{lll}K_{\operatorname{dim}}\left[\mathrm{M}^{-1}\right]= & 3200 \pm 300 \\ \Delta \delta_{\max }\left(\mathrm{H}^{\mathrm{a}}\right)[\mathrm{ppm}] & = & 0.9 \\ \Delta \delta_{\max }\left(\mathrm{H}^{\mathrm{a}^{\prime}}\right)[\mathrm{ppm}] & = & 0.7 \\ \Delta \delta_{\max }\left(\mathrm{H}^{\mathrm{b}}\right)[\mathrm{ppm}] & = & 2.8 \\ \Delta \delta_{\max }\left(\mathrm{H}^{\mathrm{b}^{\prime}}\right)[\mathrm{ppm}] & = & 2.6\end{array}$


Table S11: ${ }^{1} \mathrm{H}$ NMR dilution titration experiments at variable temperatures to determine the association constants $K_{\mathrm{dim}}$ and the differences in the ${ }^{1} \mathrm{H}$ NMR chemical shifts for the equilibrium $2 \cdot \mathbf{4 a} \leftrightarrows[\mathbf{4 a}]_{2}$ in $\mathrm{D}_{2} \mathrm{O}$

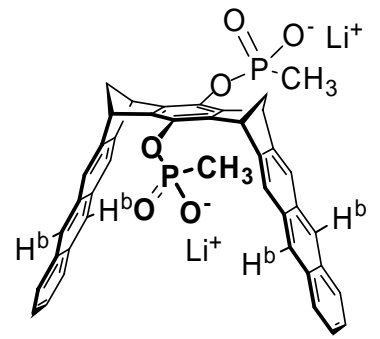

$\delta$ (monomer) $\mathrm{H}^{\mathrm{b}}[\mathrm{ppm}]=8.1\left(\mathrm{CD}_{3} \mathrm{OD}\right)$
$M_{\mathrm{R}}\left[\mathrm{g} \mathrm{mol}^{-1}\right]:$

706.53

$m_{\mathrm{R}}[\mathrm{mg}]:$

$V_{0}[\mathrm{~mL}]$ :

3.00

$[\mathbf{4 a}]_{0}[\mathrm{mM}]:$

2.60

* The $\delta$ value observed in the ${ }^{1} \mathrm{H}$ NMR spectrum of $\mathbf{4 a}$ in $\mathrm{CD}_{3} \mathrm{OD}$ is used for $\delta$ (monomer).

$T[\mathrm{~K}]: 298$

\begin{tabular}{cccc}
\hline$[\mathbf{4 a}]_{0}[\mathrm{M}]$ & $\delta_{\text {obs }}\left(\mathrm{H}^{\mathrm{b}}\right)[\mathrm{ppm}]$ & $\Delta \delta_{\text {obs }}\left(\mathrm{H}^{\mathrm{b}}\right)[\mathrm{ppm}]$ & $\Delta \delta_{\text {calc }}\left(\mathrm{H}^{\mathrm{b}}\right)[\mathrm{ppm}]$ \\
\hline 0.0026 & 5.713 & 2.367 & 2.366 \\
0.0013 & 5.747 & 2.333 & 2.335 \\
0.0007 & 5.786 & 2.294 & 2.293 \\
\hline
\end{tabular}

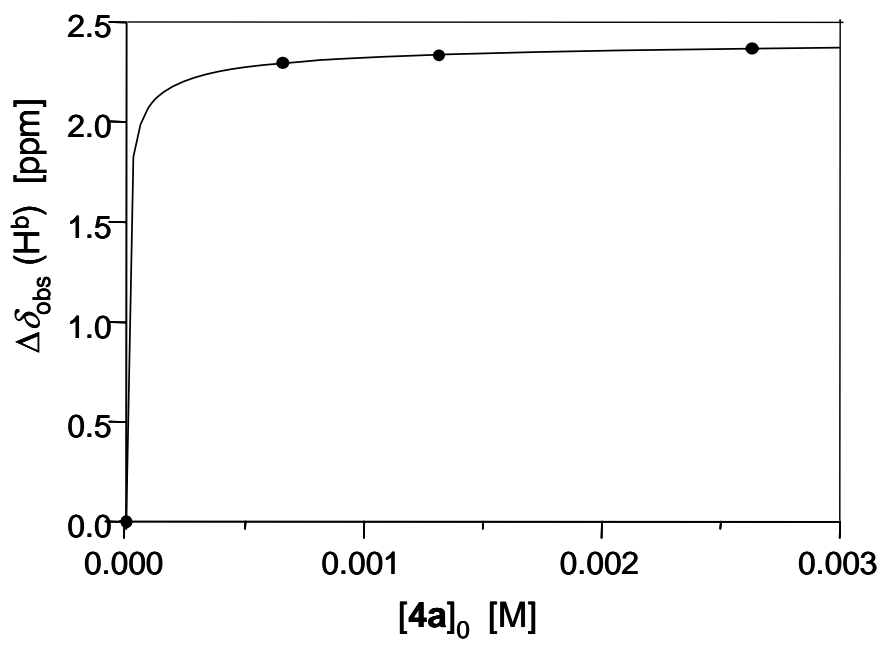

$K_{\operatorname{dim}}\left[\mathrm{M}^{-1}\right] \quad=195200 \pm 41000$

$\Delta \delta_{\max }\left(\mathrm{H}^{\mathrm{b}}\right)[\mathrm{ppm}]=$ 
$T$ [K]: 323

\begin{tabular}{cccc}
\hline$[\mathbf{4 a}]_{0}[\mathrm{M}]$ & $\delta_{\text {obs }}\left(\mathrm{H}^{\mathrm{b}}\right)[\mathrm{ppm}]$ & $\Delta \delta_{\text {obs }}\left(\mathrm{H}^{\mathrm{b}}\right)[\mathrm{ppm}]$ & $\Delta \delta_{\text {calc }}\left(\mathrm{H}^{\mathrm{b}}\right)[\mathrm{ppm}]$ \\
\hline 0.0026 & 5.831 & 2.249 & 2.252 \\
0.0013 & 5.905 & 2.175 & 2.176 \\
0.0007 & 6.000 & 2.080 & 2.072 \\
0.0003 & 6.150 & 1.930 & 1.935 \\
\hline
\end{tabular}

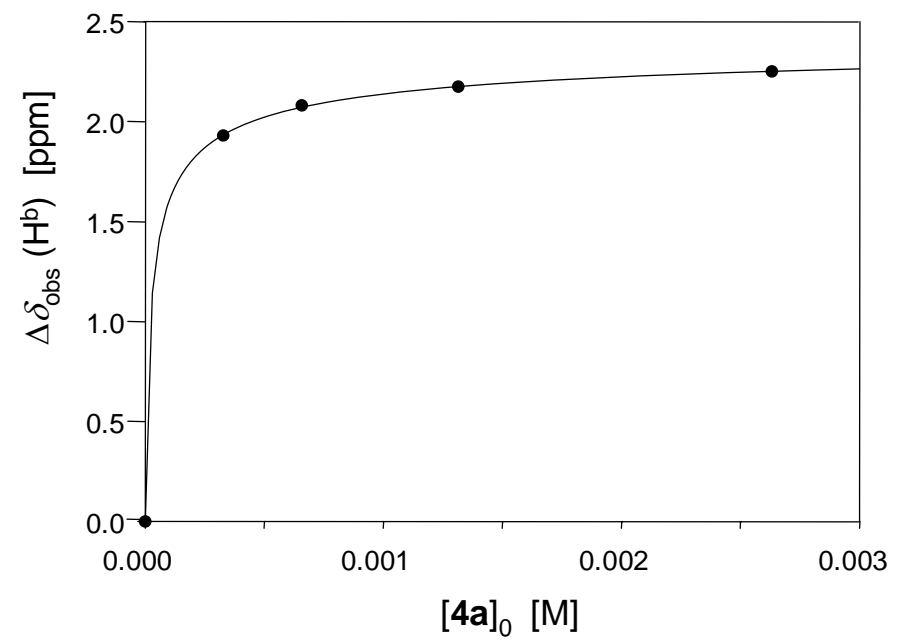

$K_{\operatorname{dim}}\left[\mathrm{M}^{-1}\right]=27300 \pm 2400$

$\Delta \delta_{\max }\left(\mathrm{H}^{\mathrm{b}}\right)[\mathrm{ppm}]=$

$T[\mathrm{~K}]: 338$

\begin{tabular}{cccc}
\hline$[\mathbf{4 a}]_{0}[\mathrm{M}]$ & $\delta_{\text {obs }}\left(\mathrm{H}^{\mathrm{b}}\right)[\mathrm{ppm}]$ & $\Delta \delta_{\text {obs }}\left(\mathrm{H}^{\mathrm{b}}\right)[\mathrm{ppm}]$ & $\Delta \delta_{\text {calc }}\left(\mathrm{H}^{\mathrm{b}}\right)[\mathrm{ppm}]$ \\
\hline 0.0026 & 5.951 & 2.129 & 2.137 \\
0.0013 & 6.063 & 2.017 & 2.018 \\
0.0007 & 6.204 & 1.876 & 1.862 \\
0.0003 & 6.415 & 1.665 & 1.662 \\
0.0002 & 6.670 & 1.410 & 1.419 \\
\hline
\end{tabular}

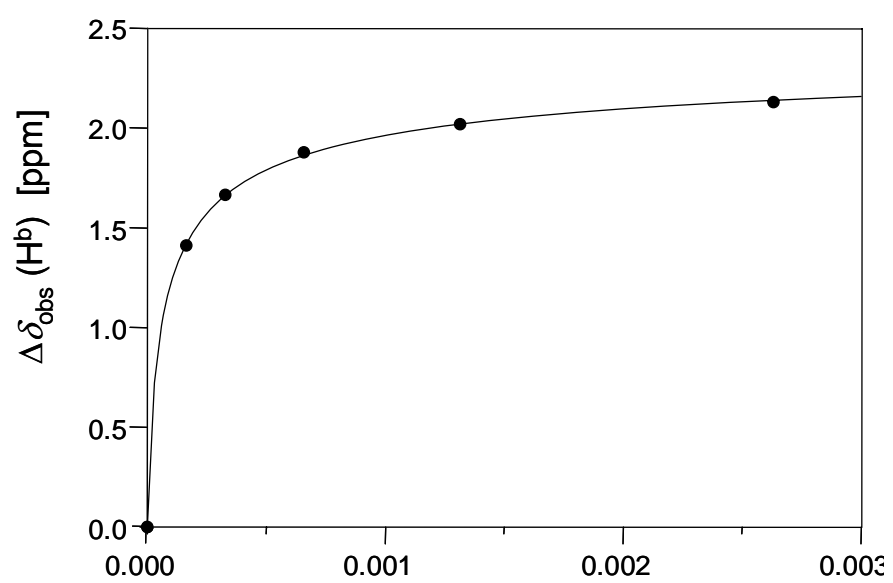

$K_{\mathrm{dim}}\left[\mathrm{M}^{-1}\right]=\quad=\quad 9800 \pm 1000$

$\Delta \delta_{\max }\left(\mathrm{H}^{\mathrm{b}}\right)[\mathrm{ppm}] \quad=\quad 2.5$

$[4 \mathrm{a}]_{0}[\mathrm{M}]$ 
$T[\mathrm{~K}]: 353$

\begin{tabular}{cccc}
\hline$[\mathbf{4 a}]_{0}[\mathrm{M}]$ & $\delta_{\text {obs }}\left(\mathrm{H}^{\mathrm{b}}\right)[\mathrm{ppm}]$ & $\Delta \delta_{\text {obs }}\left(\mathrm{H}^{\mathrm{b}}\right)[\mathrm{ppm}]$ & $\Delta \delta_{\text {calc }}\left(\mathrm{H}^{\mathrm{b}}\right)[\mathrm{ppm}]$ \\
\hline 0.0026 & 6.114 & 1.966 & 1.975 \\
0.0013 & 6.271 & 1.809 & 1.809 \\
0.0007 & 6.468 & 1.612 & 1.600 \\
0.0003 & 6.719 & 1.361 & 1.349 \\
0.0002 & 7.030 & 1.050 & 1.066 \\
\hline
\end{tabular}

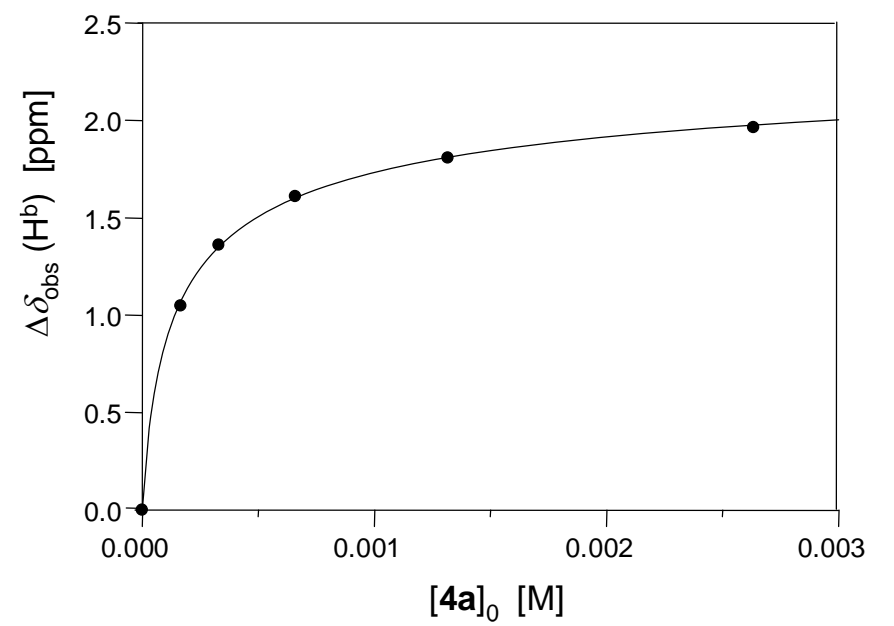

$$
\begin{aligned}
& K_{\operatorname{dim}}\left[\mathrm{M}^{-1}\right]=\quad=\quad 4200 \pm 400 \\
& \Delta \delta_{\max }\left(\mathrm{H}^{\mathrm{b}}\right)[\mathrm{ppm}] \quad=\quad 2.4
\end{aligned}
$$

$T$ [K]: 368

\begin{tabular}{cccc}
\hline$[\mathbf{4 a}]_{0}[\mathrm{M}]$ & $\delta_{\text {obs }}\left(\mathrm{H}^{\mathrm{b}}\right)[\mathrm{ppm}]$ & $\Delta \delta_{\text {obs }}\left(\mathrm{H}^{\mathrm{b}}\right)[\mathrm{ppm}]$ & $\Delta \delta_{\text {calc }}\left(\mathrm{H}^{\mathrm{b}}\right)[\mathrm{ppm}]$ \\
\hline 0.0026 & 6.360 & 1.720 & 1.715 \\
0.0013 & 6.575 & 1.505 & 1.510 \\
0.0007 & 6.819 & 1.261 & 1.266 \\
0.0003 & 7.080 & 1.000 & 0.994 \\
\hline
\end{tabular}

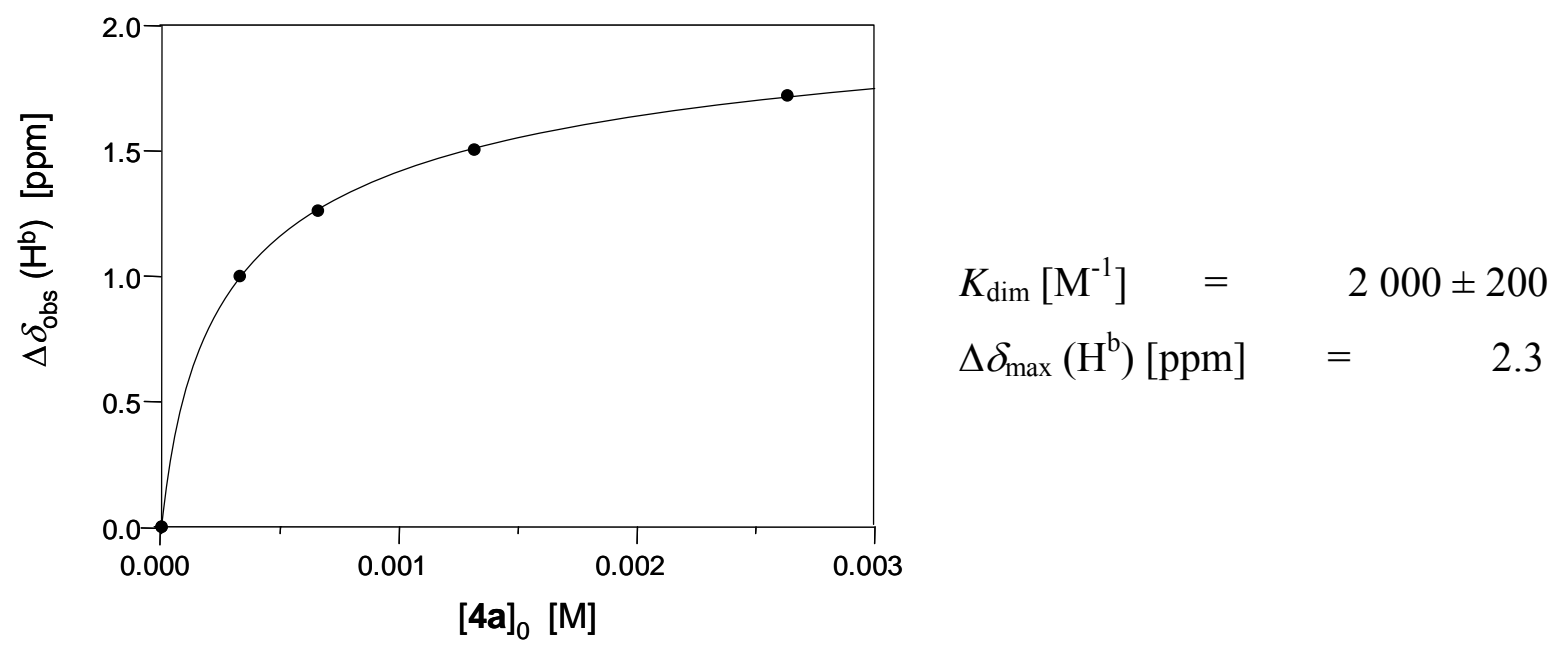


Table S12: ${ }^{1} \mathrm{H}$ NMR dilution titration experiment to determine the association constant $K_{\mathrm{a}}$ and the differences in the ${ }^{1} \mathrm{H}$ NMR chemical shifts for the equilibrium $\mathbf{2 a}+\mathbf{8} \leftrightarrows$ [2a•8] in $\mathrm{CD}_{3} \mathrm{OD}$

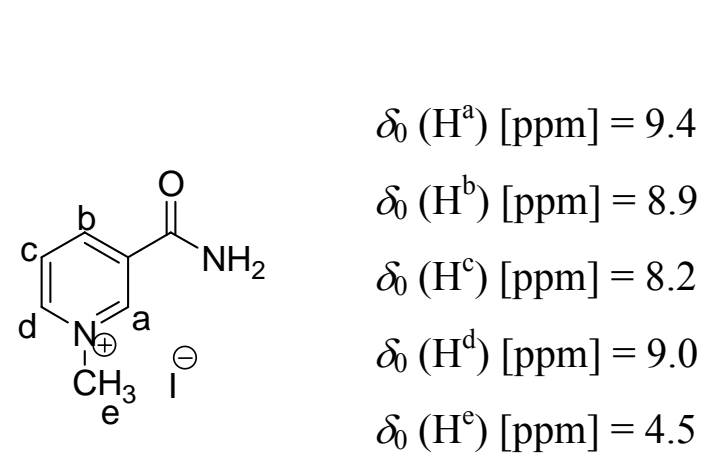

$\begin{array}{lr}M_{\mathrm{R}}(\mathbf{2 a})\left[\mathrm{g} \mathrm{mol}^{-1}\right] & 784.64 \\ M_{\mathrm{S}}(\mathbf{8}) \quad\left[\mathrm{g} \mathrm{mol}^{-1}\right] & 264.07 \\ m_{\mathrm{R}}(\mathbf{2 a})[\mathrm{mg}] & 4.07 \\ m_{\mathrm{S}}(\mathbf{8}) \quad[\mathrm{mg}] & 2.49 \\ V_{0}[\mathrm{~mL}] & 1.00 \\ {[\mathbf{2 a}]_{0}[\mathrm{mM}]} & 5.19 \\ {[\mathbf{8}]_{0} \quad[\mathrm{mM}]} & 9.43\end{array}$

$T[\mathrm{~K}]: 298$

\begin{tabular}{ccccc}
\hline$[2 \mathbf{a}]_{0}[\mathrm{M}]$ & {$[\mathbf{8}]_{0}[\mathrm{M}]$} & $\delta_{\text {obs }}\left(\mathrm{H}^{\mathrm{e}}\right)$ & $\Delta \delta_{\text {obs }}\left(\mathrm{H}^{\mathrm{e}}\right)$ & $\Delta \delta_{\text {calc }}\left(\mathrm{H}^{\mathrm{e}}\right)$ \\
\hline 0.00040 & 0.00046 & 2.992 & 1.488 & 1.478 \\
0.00034 & 0.00039 & 3.015 & 1.465 & 1.472 \\
0.00023 & 0.00026 & 3.041 & 1.438 & 1.452 \\
0.00014 & 0.00016 & 3.062 & 1.418 & 1.417 \\
0.00010 & 0.00011 & 3.099 & 1.381 & 1.382 \\
0.00007 & 0.00008 & 3.134 & 1.346 & 1.348 \\
\hline
\end{tabular}

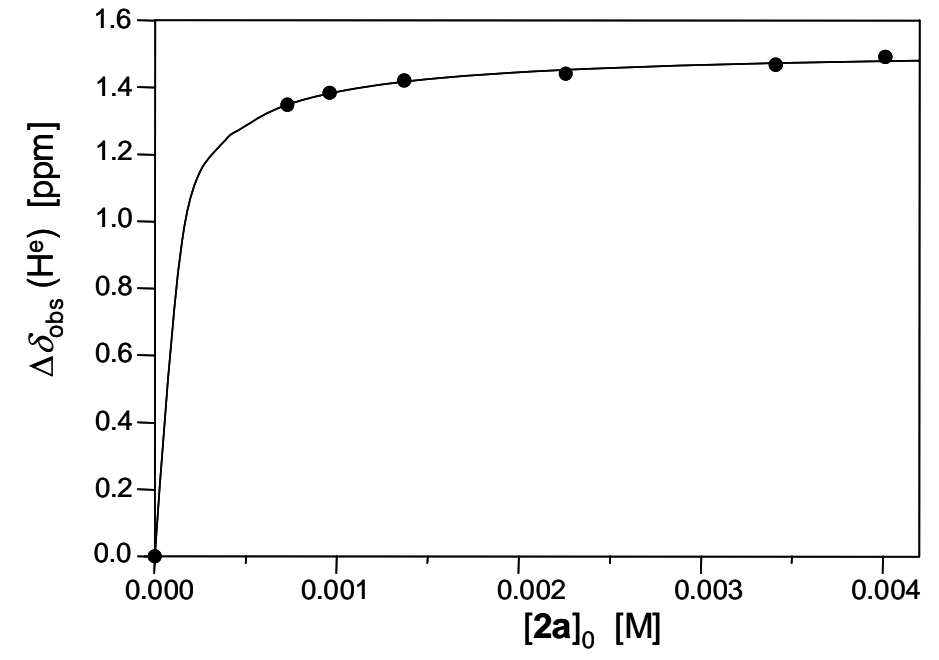

$K_{\mathrm{a}}\left[\mathrm{M}^{-1}\right]=119300 \pm 10900$

$\Delta \delta_{\max }\left(\mathrm{H}^{\mathrm{a}}\right)[\mathrm{ppm}] \quad=\quad 3.2$

$\Delta \delta_{\max }\left(\mathrm{H}^{\mathrm{b}}\right)[\mathrm{ppm}] \quad=\quad 1.5$

$\Delta \delta_{\max }\left(\mathrm{H}^{\mathrm{c}}\right)[\mathrm{ppm}] \quad=\quad 4.8$

$\Delta \delta_{\max }\left(\mathrm{H}^{\mathrm{d}}\right)[\mathrm{ppm}] \quad=\quad 2.1$

$\Delta \delta_{\max }\left(\mathrm{H}^{\mathrm{e}}\right)[\mathrm{ppm}] \quad=\quad 2.8$ 
Table S13: ${ }^{1} \mathrm{H}$ NMR dilution titration experiments at variable temperatures to determine the association constants $K_{\mathrm{a}}$ and the differences in the ${ }^{1} \mathrm{H}$ NMR chemical shifts for the equilibrium $[\mathbf{2 a}]_{2}+2 \cdot \mathbf{8} \leftrightarrows 2 \cdot[\mathbf{2 a \bullet 8}]$ in $\mathrm{D}_{2} \mathrm{O}$

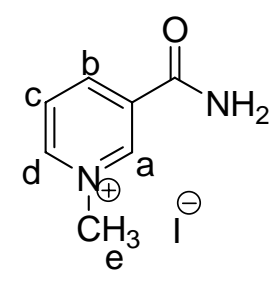

$\begin{array}{lr}M_{\mathrm{R}}(\mathbf{2 a})\left[\mathrm{g} \mathrm{mol}^{-1}\right] & 784.64 \\ M_{\mathrm{S}}(\mathbf{8}) \quad\left[\mathrm{g} \mathrm{mol}^{-1}\right] & 264.07 \\ m_{\mathrm{R}}(\mathbf{2 a})[\mathrm{mg}] & 6.19 \\ m_{\mathrm{S}}(\mathbf{8}) \quad[\mathrm{mg}] & 2.07 \\ V_{0}[\mathrm{~mL}] & 1.00 \\ {[\mathbf{2 a}]_{0}[\mathrm{mM}]} & 7.90 \\ {[\mathbf{8}]_{0} \quad[\mathrm{mM}]} & 7.84\end{array}$

$T[\mathrm{~K}]: 338 \mathrm{~K}$

$\delta_{0}[\mathrm{ppm}]=9.2\left(\mathrm{H}^{\mathrm{a}}\right), 8.8\left(\mathrm{H}^{\mathrm{b}}\right), 8.1\left(\mathrm{H}^{\mathrm{c}}\right), 8.9\left(\mathrm{H}^{\mathrm{d}}\right), 4.4\left(\mathrm{H}^{\mathrm{e}}\right)$

\begin{tabular}{ccccc}
\hline$[\mathbf{2 a}]_{0}[\mathrm{M}]$ & {$[\mathbf{8}]_{0}[\mathrm{M}]$} & $\delta_{\mathrm{S}}\left(\mathrm{H}^{\mathrm{e}}\right)$ & $\Delta \delta_{\text {obs }}\left(\mathrm{H}^{\mathrm{e}}\right)$ & $\Delta \delta_{\text {calc }}\left(\mathrm{H}^{\mathrm{e}}\right)$ \\
\hline 0.00395 & 0.00392 & 3.199 & 1.284 & 1.272 \\
0.00198 & 0.00196 & 3.266 & 1.218 & 1.218 \\
0.00132 & 0.00131 & 3.312 & 1.171 & 1.178 \\
0.00066 & 0.00065 & 3.394 & 1.089 & 1.093 \\
0.00040 & 0.00039 & 3.478 & 1.006 & 1.015 \\
0.00013 & 0.00013 & 3.673 & 0.810 & 0.800 \\
\hline
\end{tabular}

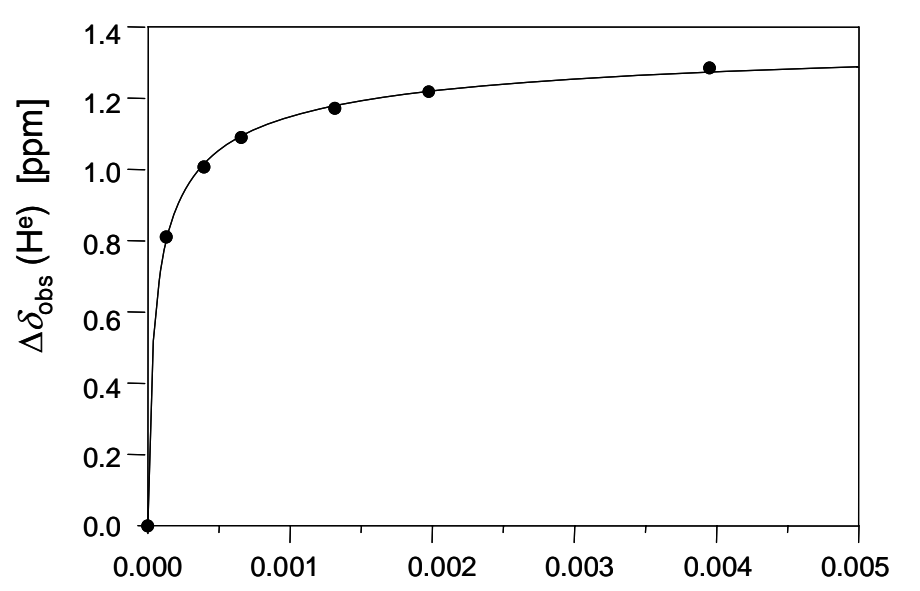

$[2 \mathrm{a}]_{0}[\mathrm{M}]$

$$
\begin{array}{lll}
K_{\mathrm{a}}\left[\mathrm{M}^{-1}\right]=23000 \pm 2300 \\
\Delta \delta_{\max }\left(\mathrm{H}_{\mathrm{a}}\right)[\mathrm{ppm}]= & \text { n.d. } \\
\Delta \delta_{\max }\left(\mathrm{H}_{\mathrm{b}}\right)[\mathrm{ppm}]= & 1.3 \\
\Delta \delta_{\max }\left(\mathrm{H}_{\mathrm{c}}\right)[\mathrm{ppm}]= & \text { n.d. } \\
\Delta \delta_{\max }\left(\mathrm{H}_{\mathrm{d}}\right)[\mathrm{ppm}]= & 1.6 \\
\Delta \delta_{\max }\left(\mathrm{H}_{\mathrm{e}}\right)[\mathrm{ppm}]= & 1.4
\end{array}
$$


$T[\mathrm{~K}]: 348$

$\delta_{0}[\mathrm{ppm}]=9.1\left(\mathrm{H}^{\mathrm{a}}\right), 8.8\left(\mathrm{H}^{\mathrm{b}}\right), 8.1\left(\mathrm{H}^{\mathrm{c}}\right), 8.8\left(\mathrm{H}^{\mathrm{d}}\right), 4.4\left(\mathrm{H}^{\mathrm{e}}\right)$

\begin{tabular}{ccccc}
\hline$[\mathbf{2 a}]_{0}[\mathrm{M}]$ & {$[\mathbf{8}]_{0}[\mathrm{M}]$} & $\delta_{\mathrm{S}}\left(\mathrm{H}^{\mathrm{e}}\right)$ & $\Delta \delta_{\text {obs }}\left(\mathrm{H}^{\mathrm{e}}\right)$ & $\Delta \delta_{\text {calc }}\left(\mathrm{H}^{\mathrm{e}}\right)$ \\
\hline 0.00395 & 0.00392 & 3.212 & 1.269 & 1.260 \\
0.00198 & 0.00196 & 3.283 & 1.198 & 1.200 \\
0.00132 & 0.00131 & 3.333 & 1.148 & 1.155 \\
0.00066 & 0.00065 & 3.420 & 1.062 & 1.061 \\
0.00040 & 0.00039 & 3.512 & 0.970 & 0.975 \\
0.00013 & 0.00013 & 3.730 & 0.751 & 0.746 \\
\hline
\end{tabular}

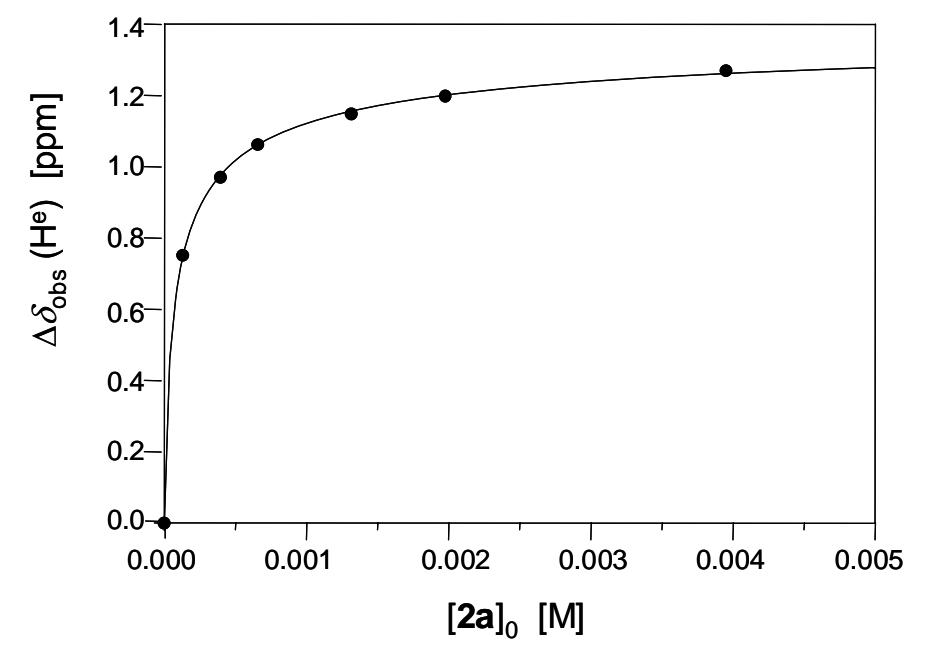

$$
\begin{array}{lcc}
K_{\mathrm{a}}\left[\mathrm{M}^{-1}\right]=17700 \pm 1800 \\
\Delta \delta_{\max }\left(\mathrm{H}_{\mathrm{a}}\right)[\mathrm{ppm}]= & \text { n.d. } \\
\Delta \delta_{\max }\left(\mathrm{H}_{\mathrm{b}}\right)[\mathrm{ppm}]= & 1.3 \\
\Delta \delta_{\max }\left(\mathrm{H}_{\mathrm{c}}\right)[\mathrm{ppm}]= & \text { n.d. } \\
\Delta \delta_{\max }\left(\mathrm{H}_{\mathrm{d}}\right)[\mathrm{ppm}]= & 1.6 \\
\Delta \delta_{\max }\left(\mathrm{H}_{\mathrm{e}}\right)[\mathrm{ppm}]= & 1.4
\end{array}
$$

$T[\mathrm{~K}]: 358$

$\delta_{0}[\mathrm{ppm}]=9.1\left(\mathrm{H}^{\mathrm{a}}\right), 8.8\left(\mathrm{H}^{\mathrm{b}}\right), 8.1\left(\mathrm{H}^{\mathrm{c}}\right), 8.9\left(\mathrm{H}^{\mathrm{d}}\right), 4.4\left(\mathrm{H}^{\mathrm{e}}\right)$

\begin{tabular}{ccccc}
\hline$[\mathbf{2 a}]_{0}[\mathrm{M}]$ & {$[\mathbf{8}]_{0}[\mathrm{M}]$} & $\delta_{\text {obs }}\left(\mathrm{H}^{\mathrm{e}}\right)$ & $\Delta \delta_{\text {obs }}\left(\mathrm{H}^{\mathrm{e}}\right)$ & $\Delta \delta_{\text {calc }}\left(\mathrm{H}^{\mathrm{e}}\right)$ \\
\hline 0.00395 & 0.00392 & 3.223 & 1.256 & 1.253 \\
0.00198 & 0.00196 & 3.298 & 1.181 & 1.183 \\
0.00132 & 0.00131 & 3.351 & 1.128 & 1.133 \\
0.00066 & 0.00065 & 3.448 & 1.031 & 1.027 \\
0.00040 & 0.00039 & 3.548 & 0.931 & 0.933 \\
0.00013 & 0.00013 & 3.788 & 0.691 & 0.687 \\
\hline
\end{tabular}

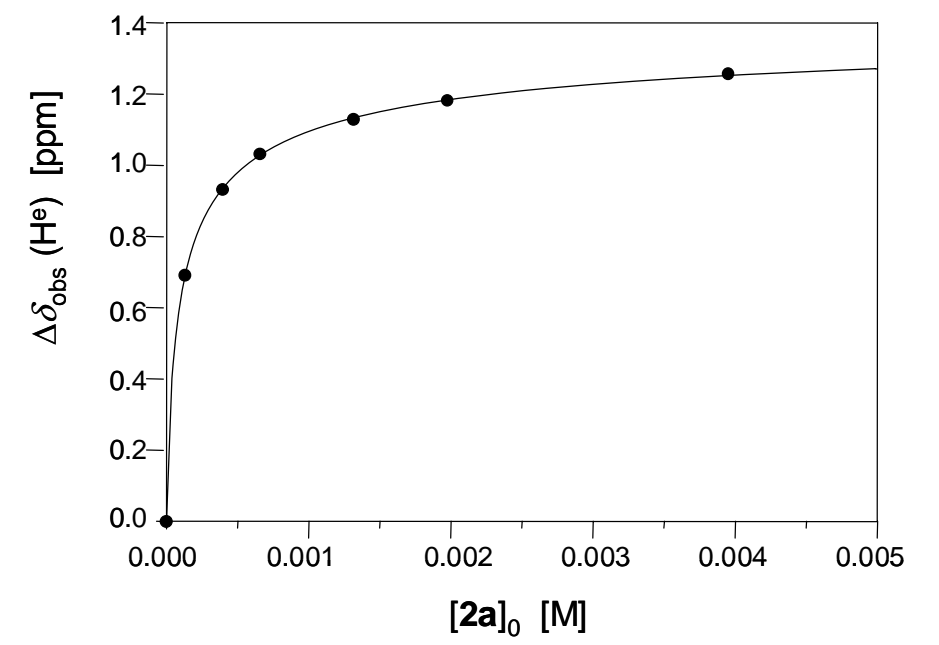

$K_{\mathrm{a}}\left[\mathrm{M}^{-1}\right] \quad=13400 \pm 1300$

$\Delta \delta_{\max }\left(\mathrm{H}_{\mathrm{a}}\right)[\mathrm{ppm}]=$ n.d.

$\Delta \delta_{\max }\left(\mathrm{H}_{\mathrm{b}}\right)[\mathrm{ppm}]=1.3$

$\Delta \delta_{\max }\left(\mathrm{H}_{\mathrm{c}}\right)[\mathrm{ppm}]=$ n.d.

$\Delta \delta_{\max }\left(\mathrm{H}_{\mathrm{d}}\right)[\mathrm{ppm}]=1.6$

$\Delta \delta_{\max }\left(\mathrm{H}_{\mathrm{e}}\right)[\mathrm{ppm}] \quad=\quad 1.4$

$[2 \mathrm{a}]_{0}[\mathrm{M}]$ 
$T[\mathrm{~K}]: 368$

$\delta_{0}[\mathrm{ppm}]=9.1\left(\mathrm{H}^{\mathrm{a}}\right), 8.8\left(\mathrm{H}^{\mathrm{b}}\right), 8.1\left(\mathrm{H}^{\mathrm{c}}\right), 8.8\left(\mathrm{H}^{\mathrm{d}}\right), 4.4\left(\mathrm{H}^{\mathrm{e}}\right)$

\begin{tabular}{ccccc}
\hline$[\mathbf{2 a}]_{0}[\mathrm{M}]$ & {$[\mathbf{8}]_{0}[\mathrm{M}]$} & $\delta_{\text {obs }}\left(\mathrm{H}^{\mathrm{e}}\right)$ & $\Delta \delta_{\text {obs }}\left(\mathrm{H}^{\mathrm{e}}\right)$ & $\Delta \delta_{\text {calc }}\left(\mathrm{H}^{\mathrm{e}}\right)$ \\
\hline 0.00395 & 0.00392 & 3.233 & 1.247 & 1.246 \\
0.00198 & 0.00196 & 3.313 & 1.167 & 1.167 \\
0.00132 & 0.00131 & 3.374 & 1.106 & 1.111 \\
0.00066 & 0.00065 & 3.480 & 0.999 & 0.993 \\
0.00040 & 0.00039 & 3.591 & 0.889 & 0.889 \\
0.00013 & 0.00013 & 3.852 & 0.628 & 0.692 \\
\hline
\end{tabular}

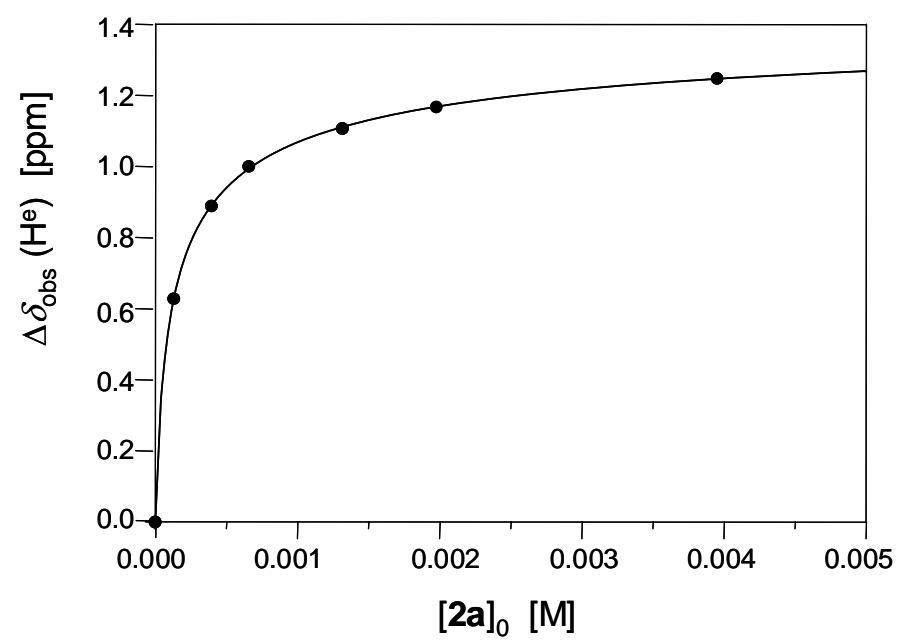

$$
\begin{array}{lrl}
K_{\mathrm{a}}\left[\mathrm{M}^{-1}\right]=10 & 200 \pm 1200 \\
\Delta \delta_{\max }\left(\mathrm{H}_{\mathrm{a}}\right)[\mathrm{ppm}]= & \text { n.d. } \\
\Delta \delta_{\max }\left(\mathrm{H}_{\mathrm{b}}\right)[\mathrm{ppm}]= & 1.3 \\
\Delta \delta_{\max }\left(\mathrm{H}_{\mathrm{c}}\right)[\mathrm{ppm}]= & \text { n.d. } \\
\Delta \delta_{\max }\left(\mathrm{H}_{\mathrm{d}}\right)[\mathrm{ppm}]= & 1.6 \\
\Delta \delta_{\max }\left(\mathrm{H}_{\mathrm{e}}\right)[\mathrm{ppm}]= & 1.5
\end{array}
$$

$[2 \mathrm{a}]_{0}[\mathrm{M}]$ 
Table S14: ${ }^{1} \mathrm{H}$ NMR dilution titration experiment to determine the association constant $K_{\mathrm{a}}$ and the differences in the ${ }^{1} \mathrm{H}$ NMR chemical shifts for the equilibrium $4 \mathbf{a}+\mathbf{8} \leftrightarrows[\mathbf{4 a \bullet 8}]$ in $\mathrm{CD}_{3} \mathrm{OD}$

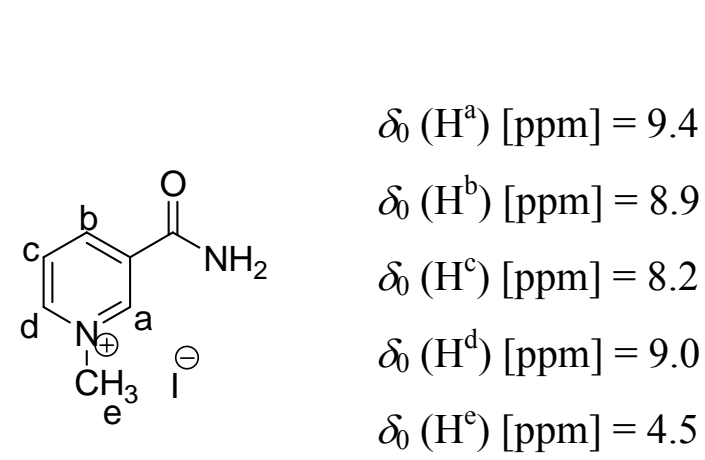

\begin{tabular}{|c|c|}
\hline$M_{\mathrm{R}}(\mathbf{4 a})\left[\mathrm{g} \mathrm{mol}^{-1}\right]$ & 706.53 \\
\hline$M_{\mathrm{S}}(\mathbf{8}) \quad\left[\mathrm{g} \mathrm{mol}^{-1}\right]$ & 264.07 \\
\hline$m_{\mathrm{R}}(\mathbf{4 a})[\mathrm{mg}]$ & 6.14 \\
\hline$m_{\mathrm{S}}(\mathbf{8}) \quad[\mathrm{mg}]$ & 2.27 \\
\hline$V_{0}[\mathrm{~mL}]$ & 3.00 \\
\hline$[4 \mathbf{a}]_{0}[\mathrm{mM}]$ & 2.90 \\
\hline$[8]_{0}[\mathrm{mM}]$ & 2.87 \\
\hline
\end{tabular}

$T[\mathrm{~K}]: 298$

\begin{tabular}{ccccc}
\hline$[\mathbf{4 a}]_{0}[\mathrm{M}]$ & {$[\mathbf{8}]_{0}[\mathrm{M}]$} & $\delta_{\text {obs }}\left(\mathrm{H}^{\mathrm{c}}\right)$ & $\Delta \delta_{\text {obs }}\left(\mathrm{H}^{\mathrm{c}}\right)$ & $\Delta \delta_{\text {calc }}\left(\mathrm{H}^{\mathrm{c}}\right)$ \\
\hline 0.0029 & 0.0029 & 6.175 & 2.017 & 1.993 \\
0.0014 & 0.0014 & 6.356 & 1.835 & 1.849 \\
0.0007 & 0.0007 & 6.549 & 1.642 & 1.664 \\
0.0004 & 0.0004 & 6.749 & 1.442 & 1.435 \\
0.0002 & 0.0002 & 7.022 & 1.169 & 1.171 \\
0.0001 & 0.0001 & 7.294 & 0.897 & 0.888 \\
\hline
\end{tabular}

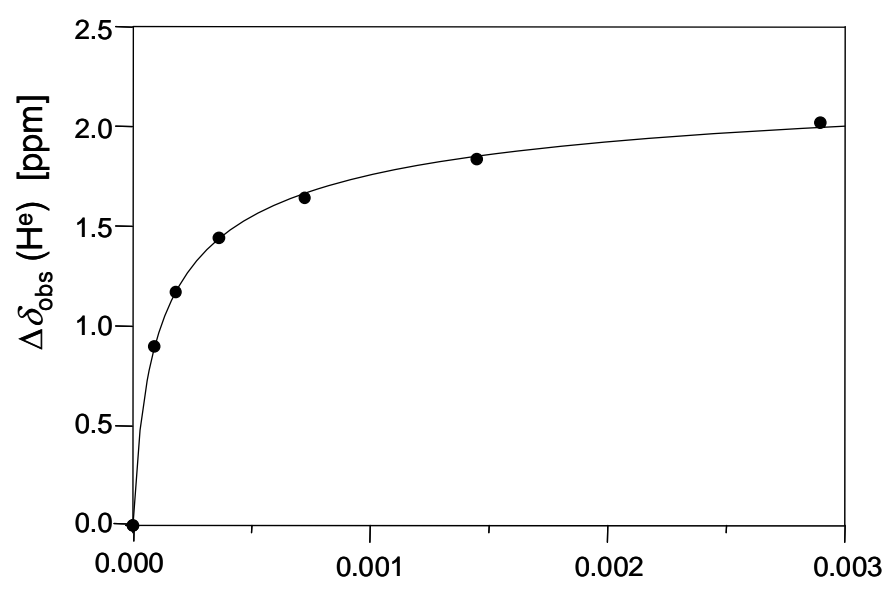

$[4 \mathrm{a}]_{0}[\mathrm{M}]$

$\begin{array}{lll}K_{\mathrm{a}}\left[\mathrm{M}^{-1}\right]= & 10400 \pm 1 & 400 \\ \Delta \delta_{\max }\left(\mathrm{H}^{\mathrm{a}}\right)[\mathrm{ppm}] & = & 1.7 \\ \Delta \delta_{\max }\left(\mathrm{H}^{\mathrm{b}}\right)[\mathrm{ppm}] & = & 2.2 \\ \Delta \delta_{\max }\left(\mathrm{H}^{\mathrm{c}}\right)[\mathrm{ppm}] & = & 2.4 \\ \Delta \delta_{\max }\left(\mathrm{H}^{\mathrm{d}}\right)[\mathrm{ppm}] & = & 1.6 \\ \Delta \delta_{\max }\left(\mathrm{H}^{\mathrm{e}}\right)[\mathrm{ppm}] & = & 1.6\end{array}$


Table S15: ${ }^{1} \mathrm{H}$ NMR dilution titration experiments at variable temperatures to determine the association constants $K_{\mathrm{a}}$ and the differences in the ${ }^{1} \mathrm{H}$ NMR chemical shifts for the equilibrium $[\mathbf{4 a}]_{2}+2 \cdot \mathbf{8} \leftrightarrows 2 \cdot[\mathbf{4 a \bullet 8}]$ in $\mathrm{D}_{2} \mathrm{O}$

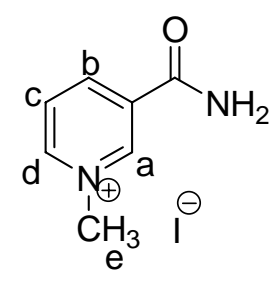

$\begin{array}{lr}M_{\mathrm{R}}(\mathbf{4 a})\left[\mathrm{g} \mathrm{mol}^{-1}\right] & 784.64 \\ M_{\mathrm{S}}(\mathbf{8}) \quad\left[\mathrm{g} \mathrm{mol}^{-1}\right] & 264.07 \\ m_{\mathrm{R}}(\mathbf{4 a})[\mathrm{mg}] & 6.19 \\ m_{\mathrm{S}}(\mathbf{8}) \quad[\mathrm{mg}] & 2.07 \\ V_{0}[\mathrm{~mL}] & 1.00 \\ {[4 \mathbf{4}]_{0}[\mathrm{mM}]} & 7.90 \\ {[\mathbf{8}]_{0} \quad[\mathrm{mM}]} & 7.84\end{array}$

$T[\mathrm{~K}]: 318 \mathrm{~K}$

$\delta_{0}[\mathrm{ppm}]=9.2\left(\mathrm{H}^{\mathrm{a}}\right), 8.8\left(\mathrm{H}^{\mathrm{b}}\right), 8.1\left(\mathrm{H}^{\mathrm{c}}\right), 8.9\left(\mathrm{H}^{\mathrm{d}}\right), 4.4\left(\mathrm{H}^{\mathrm{e}}\right)$

\begin{tabular}{ccccc}
\hline$[\mathbf{4 a}]_{0}[\mathrm{M}]$ & {$[\mathbf{8}]_{0}[\mathrm{M}]$} & $\delta_{\text {obs }}\left(\mathrm{H}^{\mathrm{e}}\right)$ & $\Delta \delta_{\text {obs }}\left(\mathrm{H}^{\mathrm{e}}\right)$ & $\Delta \delta_{\text {calc }}\left(\mathrm{H}^{\mathrm{e}}\right)$ \\
\hline 0.0022 & 0.0028 & 3.321 & 1.083 & 1.043 \\
0.0011 & 0.0014 & 3.406 & 0.998 & 1.010 \\
0.0005 & 0.0007 & 3.467 & 0.937 & 0.959 \\
0.0003 & 0.0004 & 3.550 & 0.854 & 0.886 \\
0.0001 & 0.0002 & 3.606 & 0.798 & 0.788 \\
0.0001 & 0.0001 & 3.717 & 0.687 & 0.667 \\
\hline
\end{tabular}

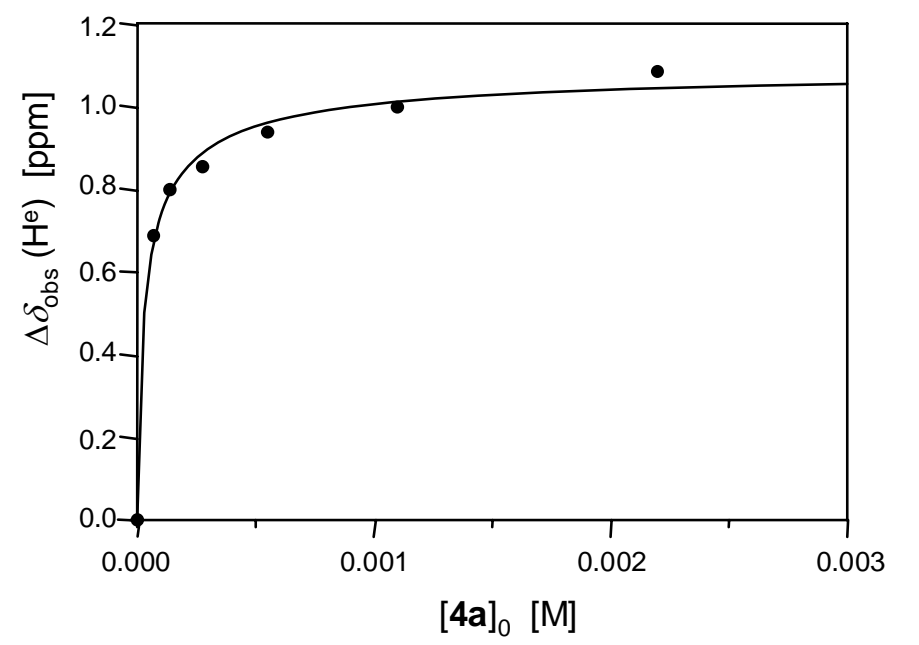

$$
\begin{array}{llc}
K_{\mathrm{a}}\left[\mathrm{M}^{-1}\right]= & 35000 \pm 6700 \\
\Delta \delta_{\max }\left(\mathrm{H}^{\mathrm{a}}\right)[\mathrm{ppm}] & = & 2.0 \\
\Delta \delta_{\max }\left(\mathrm{H}^{\mathrm{b}}\right)[\mathrm{ppm}] & = & 3.0^{*} \\
\Delta \delta_{\max }\left(\mathrm{H}^{\mathrm{c}}\right)[\mathrm{ppm}] & = & 2.4 \\
\Delta \delta_{\max }\left(\mathrm{H}^{\mathrm{d}}\right)[\mathrm{ppm}] & = & \text { n. d. } \\
\Delta \delta_{\max }\left(\mathrm{H}^{\mathrm{e}}\right)[\mathrm{ppm}] & = & 1.4
\end{array}
$$

* The broad signals in $\mathrm{D}_{2} \mathrm{O}$ could not be assigned by $\mathrm{C}, \mathrm{H}-\mathrm{COSY}$, therefore the assignment of the signals was made by comparison with the signals in $\mathrm{CD}_{3} \mathrm{OD}$. 
$T[\mathrm{~K}]: 338$

$\delta_{0}[\mathrm{ppm}]=9.2\left(\mathrm{H}^{\mathrm{a}}\right), 8.8\left(\mathrm{H}^{\mathrm{b}}\right), 8.1\left(\mathrm{H}^{\mathrm{c}}\right), 8.9\left(\mathrm{H}^{\mathrm{d}}\right), 4.4\left(\mathrm{H}^{\mathrm{e}}\right)$

\begin{tabular}{ccccc}
\hline$[\mathbf{4 a}]_{0}[\mathrm{M}]$ & {$[\mathbf{8}]_{0}[\mathrm{M}]$} & $\delta_{\text {obs }}\left(\mathrm{H}^{\mathrm{e}}\right)$ & $\Delta \delta_{\text {obs }}\left(\mathrm{H}^{\mathrm{e}}\right)$ & $\Delta \delta_{\text {calc }}\left(\mathrm{H}^{\mathrm{e}}\right)$ \\
\hline 0.0022 & 0.0028 & 3.380 & 1.015 & 0.987 \\
0.0011 & 0.0014 & 3.469 & 0.926 & 0.936 \\
0.0005 & 0.0007 & 3.548 & 0.847 & 0.864 \\
0.0003 & 0.0004 & 3.646 & 0.749 & 0.768 \\
0.0001 & 0.0002 & 3.746 & 0.649 & 0.648 \\
0.0001 & 0.0001 & 3.862 & 0.533 & 0.513 \\
\hline
\end{tabular}

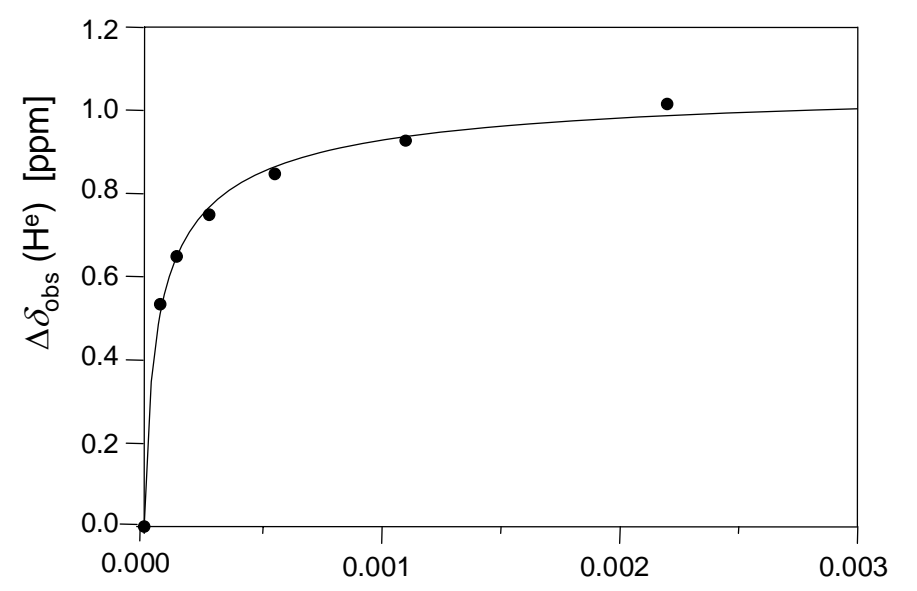

$$
\begin{array}{lcc}
K_{\mathrm{a}}\left[\mathrm{M}^{-1}\right]= & 17000 \pm 4600 \\
\Delta \delta_{\max }\left(\mathrm{H}_{\mathrm{a}}\right)[\mathrm{ppm}] & = & 1.9 \\
\Delta \delta_{\max }\left(\mathrm{H}_{\mathrm{b}}\right)[\mathrm{ppm}] & = & 2.8^{*} \\
\Delta \delta_{\max }\left(\mathrm{H}_{\mathrm{c}}\right)[\mathrm{ppm}] & = & 2.3 \\
\Delta \delta_{\max }\left(\mathrm{H}_{\mathrm{d}}\right)[\mathrm{ppm}] & = & 2.0^{*} \\
\Delta \delta_{\max }\left(\mathrm{H}_{\mathrm{e}}\right)[\mathrm{ppm}] & = & 1.4
\end{array}
$$

$[4 a]_{0}[\mathrm{M}]$

$T[\mathrm{~K}]: 358$

$\delta_{0}[\mathrm{ppm}]=9.1\left(\mathrm{H}^{\mathrm{a}}\right), 8.8\left(\mathrm{H}^{\mathrm{b}}\right), 8.1\left(\mathrm{H}^{\mathrm{c}}\right), 8.9\left(\mathrm{H}^{\mathrm{d}}\right), 4.4\left(\mathrm{H}^{\mathrm{e}}\right)$

\begin{tabular}{ccccc}
\hline$[\mathbf{4 a}]_{0}[\mathrm{M}]$ & {$[\mathbf{8}]_{0}[\mathrm{M}]$} & $\delta_{\text {obs }}\left(\mathrm{H}^{\mathrm{e}}\right)$ & $\Delta \delta_{\text {obs }}\left(\mathrm{H}^{\mathrm{e}}\right)$ & $\Delta \delta_{\text {calc }}\left(\mathrm{H}^{\mathrm{e}}\right)$ \\
\hline 0.0022 & 0.0028 & 3.458 & 0.931 & 0.916 \\
0.0011 & 0.0014 & 3.557 & 0.832 & 0.844 \\
0.0005 & 0.0007 & 3.643 & 0.746 & 0.748 \\
0.0003 & 0.0004 & 3.769 & 0.620 & 0.630 \\
0.0001 & 0.0002 & 3.892 & 0.497 & 0.496 \\
0.0001 & 0.0001 & 4.019 & 0.370 & 0.360 \\
\hline
\end{tabular}

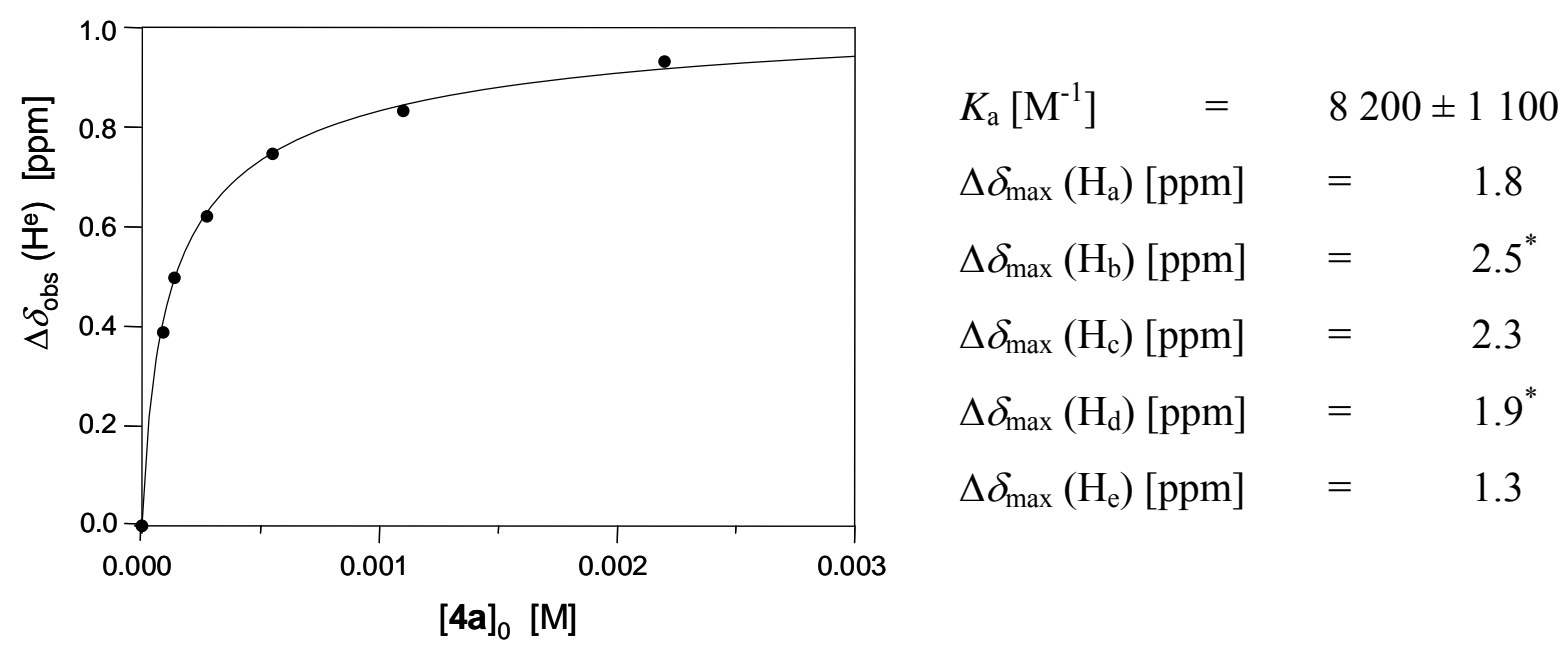

* The broad signals in $\mathrm{D}_{2} \mathrm{O}$ could not be assigned by C,H-COSY. Therefore, the assignment of the signals was made by comparison with the signals in $\mathrm{CD}_{3} \mathrm{OD}$. 
$T[\mathrm{~K}]: 368$

$\delta_{0}[\mathrm{ppm}]=9.1\left(\mathrm{H}^{\mathrm{a}}\right), 8.8\left(\mathrm{H}^{\mathrm{b}}\right), 8.1\left(\mathrm{H}^{\mathrm{c}}\right), 8.8\left(\mathrm{H}^{\mathrm{d}}\right), 4.4\left(\mathrm{H}^{\mathrm{e}}\right)$

\begin{tabular}{ccccc}
\hline$[\mathbf{4 a}]_{0}[\mathrm{M}]$ & {$[\mathbf{8}]_{0}[\mathrm{M}]$} & $\delta_{\text {obs }}\left(\mathrm{H}^{\mathrm{e}}\right)$ & $\Delta \delta_{\text {obs }}\left(\mathrm{H}^{\mathrm{e}}\right)$ & $\Delta \delta_{\text {calc }}\left(\mathrm{H}^{\mathrm{e}}\right)$ \\
\hline 0.0022 & 0.0028 & 3.521 & 0.865 & 0.848 \\
0.0011 & 0.0014 & 3.628 & 0.758 & 0.769 \\
0.0005 & 0.0007 & 3.730 & 0.656 & 0.667 \\
0.0003 & 0.0004 & 3.848 & 0.538 & 0.546 \\
0.0001 & 0.0002 & 3.968 & 0.418 & 0.415 \\
0.0001 & 0.0001 & 4.084 & 0.303 & 0.289 \\
\hline
\end{tabular}

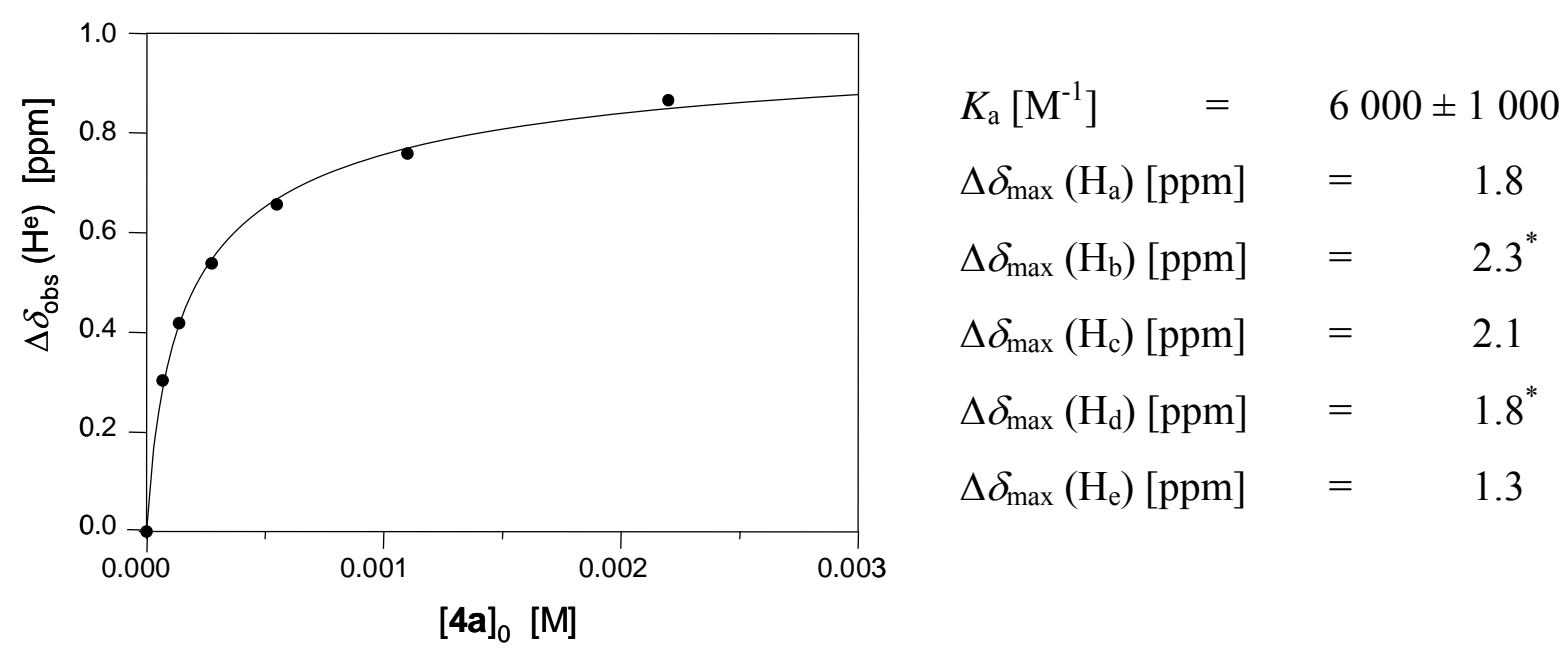

* The broad signals in $\mathrm{D}_{2} \mathrm{O}$ could not be assigned by C,H-COSY. Therefore, the assignment of the signals was made by comparison with the signals in $\mathrm{CD}_{3} \mathrm{OD}$.

Complete Ref. (38):

Kong, J.; White, C. A.; Krylov, A. I.; Sherrill, D.; Adamson, R. D.; Furlani, T. R.; Lee, M. S.; Lee, A. M.; Gwaltney, S. R.; Adams, T. R.; Ochsenfeld, C.; Gilbert, A. T. B.; Kedziora, G. S.; Rassolov, V. A.; Maurice, D. R.; Nair, N.; Shao, Y. H.; Besley, N. A.; Maslen, P. E.; Dombroski, J. P.; Daschel, H.; Zhang, W. M.; Korambath, P. P.; Baker, J.; Byrd, E. F. C.; Van Voorhis, T.; Oumi, M.; Hirata, S.; Hsu, C. P.; Ishikawa, N.; Florian, J.; Warshel, A.; Johnson, B. G.; Gill, P. M. W.; Head-Gordon, M.; Pople, J. A. J. Comput. Chem. 2000, 21, 1532-1548. 